\title{
Adaptive Control of the NASA Generic Transport Model Using Retrospective Cost Optimization
}

\begin{abstract}
Mario A. Santillo, ${ }^{*}$ Matthew S. Holzel ${ }^{\dagger}{\text { Jesse B. Hoagg },{ }^{\ddagger} \text { and Dennis S. Bernstein }}^{\S}$
We provide a detailed description of retrospective-cost based adaptive control, which is a discrete-time adaptive control law for stabilization, command following, and disturbance rejection that is effective for systems that are unstable, MIMO, and/or nonminimum phase. The adaptive control algorithm includes guidelines concerning the modeling information needed for implementation. This information includes the sign of the high-frequency gain as well as the nonminimum-phase zeros. Except when the plant has nonminimum-phase zeros whose absolute value is less than the plant's spectral radius, the required information can be approximated by a sufficient number of Markov parameters. No additional information about the poles or zeros need be known, and no matching conditions are required. We apply this adaptive control technique to NASA's Generic Transport Model to illustrate disturbance rejection under unknown, reduced controller authority.
\end{abstract}

\section{Introduction}

Unlike robust control, which chooses control gains based on a prior, fixed level of modeling uncertainty, adaptive control algorithms tune the feedback gains in response to the true plant and exogenous signals, that is, commands and disturbances. Generally speaking, adaptive controllers require less prior modeling information than robust controllers, and thus can be viewed as highly parameter-robust control laws. The price paid for the ability of adaptive control laws to operate with limited prior modeling information is the complexity of analyzing and quantifying the stability and performance of the closed-loop system, especially in light of the fact that adaptive control laws, even for linear plants, are nonlinear.

Stability and performance analysis of adaptive control laws often entails assumptions on the dynamics of the plant. For example, a widely invoked assumption in adaptive control is passivity, ${ }^{1}$ which is restrictive and difficult to verify in practice. A related assumption is that the plant is minimum phase, ${ }^{2,3}$ which may entail the same difficulties. In fact, sampling may give rise to nonminimum-phase zeros whether or not the continuous-time system is minimum phase, ${ }^{4}$ which must ultimately be accounted for by any adaptive control algorithm implemented digitally on a sampled-data control system. Beyond these assumptions, adaptive control laws are known to be sensitive to unmodeled dynamics and sensor noise, ${ }^{5,6}$ which necessitates robust adaptive control laws. ${ }^{7}$

In addition to these basic issues, adaptive control laws may entail unacceptable transients during adaptation, which may be exacerbated by actuator limitations. ${ }^{8-10}$ In fact, adaptive control under extremely limited modeling information, such as uncertainty in the sign of the high-frequency gain, ${ }^{11,12}$ may yield a transient response that exceeds the practical limits of the plant. Therefore, the type and quality of the available modeling information as well as the speed of adaptation must be considered in the analysis and implementation of adaptive control laws. These issues are stressed in. ${ }^{13}$

Adaptive control laws have been developed in both continuous-time and discrete-time settings. In the present paper we consider discrete-time adaptive control laws since these control laws can be implemented

\footnotetext{
${ }^{*}$ National Defense Science and Engineering Graduate Fellow, Department of Aerospace Engineering, The University of Michigan, Ann Arbor, MI 48109-2140, santillo@umich.edu

${ }^{\dagger}$ Graduate Research Assistant, Department of Aerospace Engineering The University of Michigan, Ann Arbor, MI 481092140, mholzel@umich.edu

$\ddagger$ Postdoctoral Research Fellow, Department of Aerospace Engineering The University of Michigan, Ann Arbor, MI 481092140, jhoagg@umich.edu

$\S$ Professor, Department of Aerospace Engineering The University of Michigan, Ann Arbor, MI 48109-2140, dsbaero@umich.edu
} 
directly in embedded code for sampled-data control systems without requiring an intermediate discretization step that may entail loss of stability margins. References on discrete-time adaptive control include..$^{2,3,14-20}$ $\mathrm{In}^{2}$ a discrete-time adaptive control law with guaranteed stability is developed under a minimum-phase assumption. Extensions given in $^{3}$ based on internal model control ${ }^{21}$ and Lyapunov analysis also invoke this assumption. To circumvent the minimum-phase assumption, the zero annihilation periodic control law ${ }^{19}$ uses lifting to move all of the plant zeros to the origin. The drawback of lifting, however, is the need for open-loop operation during alternating data windows.

Motivated by the adaptive control laws given $\mathrm{in}^{3}$ and,${ }^{20}$ the goal of the present paper is to develop a discrete-time adaptive control law that is effective for nonminimum-phase systems. The control law given $i^{20}$ lacks a proof of stability, but is known numerically to be effective on nonminimum-phase plants without recourse to lifting. Accordingly, we present an adaptive control algorithm that extends the retrospective cost optimization approach used in. ${ }^{20}$ In particular, we define an extended retrospective cost that includes control weighting as well as a learning rate, which can be used to adjust the rate of controller convergence and thus the transient behavior of the closed-loop system. Unlike, ${ }^{20}$ which uses a gradient update, the present paper uses a Newton-like update for the controller gains as the closed-form solution to a convex optimization problem. No off-line calculations are needed to implement the algorithm. A key aspect of this extension is the fact that the required modeling information is given by the Markov parameters from the control inputs to the performance variables. Except when the plant has nonminimum-phase zeros whose absolute value is less than the plant's spectral radius, we show that the relative degree, sign of the high-frequency gain, and nonminimum-phase zeros can be approximated by a sufficient number of Markov parameters. No matching conditions are required on either the plant uncertainty or the disturbances.

The goal of the present paper is to review the details of the RCF adaptive control algorithm and apply it to the NASA Generic Transport Model (GTM). ${ }^{22-24}$ We consider the problem of rejecting wind gusts under both full and limited control surface effectiveness. The controller adapts for the full 6DOF dynamics of the aircraft. All simulations use only three measurements, namely, roll angle, yaw rate, and altitude. No measurement of velocity is used by the adaptive controller.

\section{Problem Formulation}

Consider the MIMO discrete-time system

$$
\begin{aligned}
x(k+1) & =A x(k)+B u(k)+D_{1} w(k), \\
y(k) & =C x(k)+D_{2} w(k), \\
z(k) & =E_{1} x(k)+E_{0} w(k),
\end{aligned}
$$

where $x(k) \in \mathbb{R}^{n}, y(k) \in \mathbb{R}^{l_{y}}, z(k) \in \mathbb{R}^{l_{z}}, u(k) \in \mathbb{R}^{l_{u}}, w(k) \in \mathbb{R}^{l_{w}}$, and $k \geq 0$. Our goal is to develop an adaptive output feedback controller under which the performance variable $z$ is minimized in the presence of the exogenous signal $w$. In (1)-(3), w can represent either a command signal to be followed, an external disturbance to be rejected, or both. For example, if $D_{1}=0$ and $E_{0} \neq 0$, then the objective is to have the output $E_{1} x$ follow the command signal $-E_{0} w$. On the other hand, if $D_{1} \neq 0$ and $E_{0}=0$, then the objective is to reject the disturbance $w$ from the performance variable $E_{1} x$. The combined command following and disturbance rejection problem is addressed when $D_{1}$ and $E_{0}$ are suitably partitioned matrices. More precisely, if $D_{1}=\left[\begin{array}{ll}D_{11} & 0\end{array}\right], E_{0}=\left[\begin{array}{ll}0 & E_{02}\end{array}\right]$, and $w(k)=\left[\begin{array}{l}w_{1}(k) \\ w_{2}(k)\end{array}\right]$, then the objective is to have $E_{1} x$ follow the command $-E_{02} w_{2}$ while rejecting the disturbance $D_{11} w_{1}$. Lastly, if $D_{1}$ and $E_{0}$ are zero matrices, then the objective is output stabilization, that is, convergence of $z$ to zero. We assume that the open-loop system (1)-(3) is stabilizable and detectable and that measurements of $y$ and $z$ are available for feedback. If the command signal is included as a component of $y$, then the adaptive controller has a feedforward architecture. For disturbance rejection problems, the controller does not require measurements of the external disturbance $w$.

Model reference adaptive control (MRAC) is a special case of (1)-(3), where $z \triangleq y_{1}-y_{\mathrm{m}}$ is the difference between the measured output $y_{1}$ of the plant $G$ and the output $y_{\mathrm{m}}$ of a reference model $G_{\mathrm{m}}$. For MRAC, the exogenous command $w$ is assumed to be available to the controller as an additional measurement variable $y_{2}$, as shown in Figure 1. 


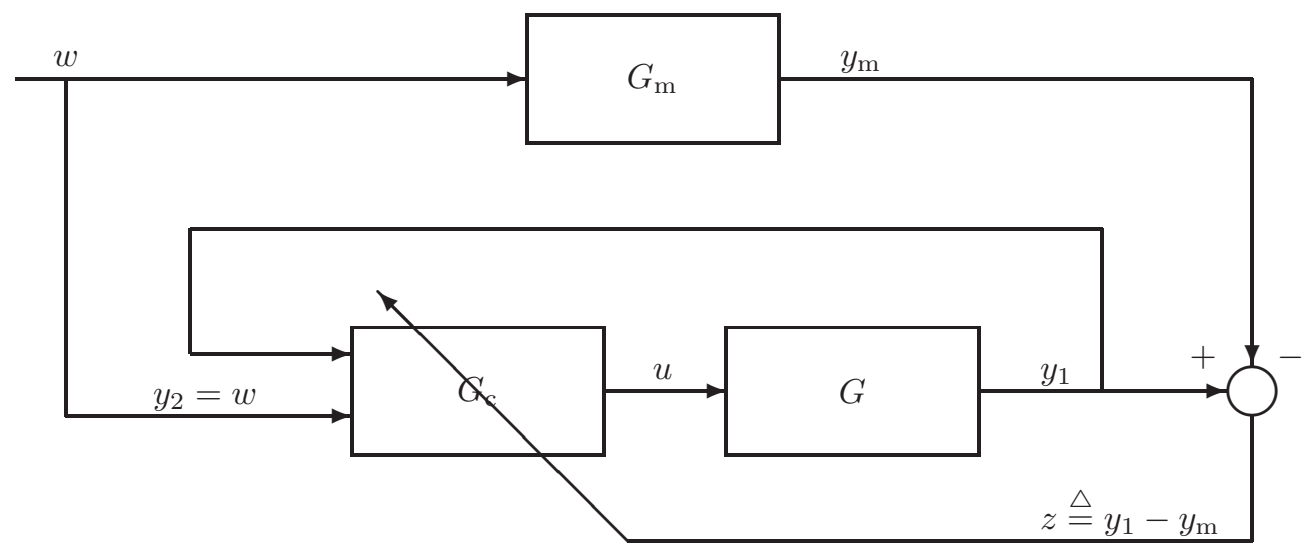

Figure 1: Model reference adaptive control problem with performance variable $z$.

\section{ARMAX Modeling}

Consider the ARMAX representation of (1), (3), given by

$$
z(k)=\sum_{i=1}^{n}-\alpha_{i} z(k-i)+\sum_{i=1}^{n} \beta_{i} u(k-i)+\sum_{i=0}^{n} \gamma_{i} w(k-i)
$$

where $\alpha_{1}, \ldots, \alpha_{n} \in \mathbb{R}, \beta_{1}, \ldots, \beta_{n} \in \mathbb{R}^{l_{z} \times l_{u}}$, and $\gamma_{0}, \ldots, \gamma_{n} \in \mathbb{R}^{l_{z} \times l_{w}}$. We define the relative degree $d \geq 1$ as the smallest positive integer $i$ such that the $i$ th Markov parameter $H_{i} \triangleq E_{1} A^{i-1} B \in \mathbb{R}^{l_{z} \times l_{u}}$ is nonzero. Note that, if $d=1$, then $H_{1}=\beta_{1}$, whereas, if $d \geq 2$, then $\beta_{1}=\ldots=\beta_{d-1}=H_{1}=\ldots=H_{d-1}=0$ and $H_{d}=\beta_{d}$.

Letting the data window size $p$ be a positive integer, we define the extended performance vector $Z(k) \in$ $\mathbb{R}^{p l_{z}}$ and $U_{1}(k) \in \mathbb{R}^{q_{\mathrm{c}} l_{u}}$ by

$$
Z(k) \triangleq\left[\begin{array}{c}
z(k) \\
\vdots \\
z(k-p+1)
\end{array}\right], \quad U_{1}(k) \triangleq\left[\begin{array}{c}
u(k-1) \\
\vdots \\
u\left(k-q_{\mathrm{c}}\right)
\end{array}\right],
$$

where $q_{\mathrm{c}} \triangleq n+p-1$. The data window size $p$ has a small but noticeable effect on transient behavior; we choose $p=1$ for all of the numerical examples in this paper. Now, (4) can be written in the form

$$
Z(k)=W_{z w} \phi_{z w}(k)+B_{z u} U_{1}(k),
$$

where

$$
\begin{aligned}
& W_{z w} \triangleq\left[\begin{array}{cccccccccccc}
-\alpha_{1} I_{l_{z}} & \cdots & -\alpha_{n} I_{l_{z}} & 0_{l_{z}} & \cdots & 0_{l_{z}} & \gamma_{0} & \cdots & \gamma_{n} & 0_{l_{z} \times l_{w}} & \cdots & 0_{l_{z} \times l_{w}} \\
0_{l_{z}} & \ddots & & \ddots & \ddots & \vdots & 0_{l_{z} \times l_{w}} & \ddots & & \ddots & \ddots & \vdots \\
\vdots & \ddots & \ddots & & \ddots & 0_{l_{z}} & \vdots & \ddots & \ddots & & \ddots & 0_{l_{z} \times l_{w}} \\
0_{l_{z}} & \cdots & 0_{l_{z}} & -\alpha_{1} I_{l_{z}} & \cdots & -\alpha_{n} I_{l_{z}} & 0_{l_{z} \times l_{w}} & \cdots & 0_{l_{z} \times l_{w}} & \gamma_{0} & \cdots & \gamma_{n}
\end{array}\right] \\
& B_{z u} \triangleq\left[\begin{array}{cccccc}
\beta_{1} & \cdots & \beta_{n} & 0_{l_{z} \times l_{u}} & \cdots & 0_{l_{z} \times l_{u}} \\
0_{l_{z} \times l_{u}} & \ddots & & \ddots & \ddots & \vdots \\
\vdots & \ddots & \ddots & & \ddots & 0_{l_{z} \times l_{u}} \\
0_{l_{z} \times l_{u}} & \cdots & 0_{l_{z} \times l_{u}} & \beta_{1} & \cdots & \beta_{n}
\end{array}\right] \in \mathbb{R}^{p l_{z} \times q_{\mathrm{c}} l_{u}},
\end{aligned}
$$


and

$$
\phi_{z w}(k) \triangleq\left[\begin{array}{c}
z(k-1) \\
\vdots \\
z(k-p-n+1) \\
w(k) \\
\vdots \\
w(k-p-n+1)
\end{array}\right] .
$$

Note that $W_{z w}$ and $B_{z u}$ have block-Toeplitz structure.

\section{Controller Construction}

To formulate an adaptive control algorithm for (1)-(3), we use a strictly proper time-series controller of order $n_{\mathrm{c}}$ such that the control $u(k)$ is given by

$$
u(k)=\sum_{i=1}^{n_{\mathrm{c}}} P_{i}(k) u(k-i)+\sum_{i=1}^{n_{\mathrm{c}}} Q_{i}(k) y(k-i)
$$

where, for all $i=1, \ldots, n_{\mathrm{c}}, P_{i}(k) \in \mathbb{R}^{l_{u} \times l_{u}}$ and $Q_{i}(k) \in \mathbb{R}^{l_{u} \times l_{y}}$. The controller order $n_{\mathrm{c}}$ is determined by standard control guidelines in terms of stabilization and disturbance rejection. The control (10) can be expressed as

$$
u(k)=\theta(k) \phi(k),
$$

where

$$
\theta(k) \triangleq\left[\begin{array}{llllll}
Q_{1}(k) & \cdots & Q_{n_{\mathrm{c}}}(k) & P_{1}(k) & \cdots & P_{n_{\mathrm{c}}}(k)
\end{array}\right] \in \mathbb{R}^{l_{u} \times n_{\mathrm{c}}\left(l_{u}+l_{y}\right)}
$$

is the controller gain matrix, and the regressor vector $\phi(k)$ is given by

$$
\phi(k) \triangleq\left[\begin{array}{c}
y(k-1) \\
\vdots \\
y\left(k-n_{\mathrm{c}}\right) \\
u(k-1) \\
\vdots \\
u\left(k-n_{\mathrm{c}}\right)
\end{array}\right] \in \mathbb{R}^{n_{\mathrm{c}}\left(l_{u}+l_{y}\right)} .
$$

We define the extended control vector $U(k) \in \mathbb{R}^{p_{c} l_{u}}$ by

$$
U(k) \triangleq\left[\begin{array}{c}
u(k-1) \\
\vdots \\
u\left(k-p_{\mathrm{c}}\right)
\end{array}\right],
$$

where $p_{\mathrm{c}} \triangleq n+r+p-2$ and $r \geq 1$. From (11), it follows that the extended control vector $U(k)$ can be written as

$$
U(k)=\sum_{i=1}^{p_{c}} L_{i} \theta(k-i) \phi(k-i)
$$

where

$$
L_{i} \triangleq\left[\begin{array}{c}
0_{(i-1) l_{u} \times l_{u}} \\
I_{l_{u}} \\
0_{\left(p_{\mathrm{c}}-i\right) l_{u} \times l_{u}}
\end{array}\right] \in \mathbb{R}^{p_{\mathrm{c}} l_{u} \times l_{u}}
$$


Next, we define the retrospective performance vector $\hat{Z}(\hat{\theta}, k) \in \mathbb{R}^{p l_{z}}$ by

$$
\hat{Z}(\hat{\theta}, k) \triangleq W_{z w} \phi_{z w}(k)+B_{z u} U_{1}(k)-\bar{B}_{z u}[U(k)-\hat{U}(\hat{\theta}, k)],
$$

where $\hat{\theta} \in \mathbb{R}^{l_{u} \times n_{\mathrm{c}}\left(l_{u}+l_{y}\right)}, \bar{B}_{z u} \in \mathbb{R}^{p l_{z} \times p_{\mathrm{c}} l_{u}}$ is the surrogate input matrix, and

$$
\hat{U}(\hat{\theta}, k) \triangleq \sum_{i=1}^{p_{c}} L_{i} \hat{\theta} \phi(k-i)
$$

is the recomputed extended control vector. Substituting (6) into (17) yields

$$
\hat{Z}(\hat{\theta}, k)=Z(k)-\bar{B}_{z u}[U(k)-\hat{U}(\hat{\theta}, k)] .
$$

The matrix $\bar{B}_{z u}$ is discussed in Section VI. Note that, since $\gamma_{0}, \ldots, \gamma_{n}$ are unknown, it follows that $W_{z w}$ is unknown, even if $w$ (and thus $\phi_{z w}$ ) is known.

Taking the vec of (19) yields

$$
\hat{Z}(\hat{\theta}, k)=f(k)+D(k) \operatorname{vec} \hat{\theta},
$$

where

$$
\begin{aligned}
f(k) \triangleq Z(k)-\bar{B}_{z u} U(k) \in \mathbb{R}^{p l_{z}}, \\
D(k) \triangleq \sum_{i=1}^{p_{\mathrm{c}}} \phi^{\mathrm{T}}(k-i) \otimes\left(\bar{B}_{z u} L_{i}\right) \in \mathbb{R}^{p l_{z} \times n_{\mathrm{c}} l_{u}\left(l_{u}+l_{y}\right)},
\end{aligned}
$$

and $\otimes$ represents the Kronecker product. Now, consider the retrospective cost function

$$
\begin{aligned}
J(\hat{\theta}, k) \triangleq & \hat{Z}^{\mathrm{T}}(\hat{\theta}, k) R_{1}(k) \hat{Z}(\hat{\theta}, k)+2 \hat{Z}^{\mathrm{T}}(\hat{\theta}, k) R_{12}(k) \hat{u}(\hat{\theta}, k+1) \\
& +\hat{u}^{\mathrm{T}}(\hat{\theta}, k+1) R_{2}(k) \hat{u}(\hat{\theta}, k+1)+\operatorname{tr}\left[R_{3}(k)(\hat{\theta}-\theta(k))^{\mathrm{T}} R_{4}(k)(\hat{\theta}-\theta(k))\right],
\end{aligned}
$$

where $R_{1}(k) \in \mathbb{R}^{p l_{z} \times p l_{z}}, R_{12}(k) \in \mathbb{R}^{p l_{z} \times l_{u}}, R_{2}(k) \in \mathbb{R}^{l_{u} \times l_{u}}, R_{3}(k) \in \mathbb{R}^{n_{\mathrm{c}}\left(l_{u}+l_{y}\right) \times n_{\mathrm{c}}\left(l_{u}+l_{y}\right)}, R_{4}(k) \in \mathbb{R}^{l_{u} \times l_{u}}$, $\left[\begin{array}{cc}R_{1}(k) & R_{12}(k) \\ R_{12}^{\mathrm{T}}(k) & R_{2}(k)\end{array}\right]$ is positive semidefinite, $R_{3}(k)$ and $R_{4}(k)$ are positive definite, and

$$
\hat{u}(\hat{\theta}, k) \triangleq \hat{\theta} \phi(k) .
$$

Substituting (20) into (23) yields

$$
J(\hat{\theta}, k)=c(k)+b^{\mathrm{T}}(k) \operatorname{vec} \hat{\theta}+(\operatorname{vec} \hat{\theta})^{\mathrm{T}} M(k) \operatorname{vec} \hat{\theta}
$$

where

$$
\begin{aligned}
& M(k) \triangleq D^{\mathrm{T}}(k) R_{1}(k) D(k)+2 D^{\mathrm{T}}(k)\left[\phi^{\mathrm{T}}(k) \otimes R_{12}(k)\right] \\
&+\left[\phi(k) \phi^{\mathrm{T}}(k)\right] \otimes R_{2}(k)+R_{3}(k) \otimes R_{4}(k), \\
& b(k) \triangleq 2 D^{\mathrm{T}}(k) R_{1}(k) f(k)+2\left[\phi(k) \otimes R_{12}^{\mathrm{T}}(k)\right] f(k)-2\left[R_{3}(k) \otimes R_{4}(k)\right] \operatorname{vec} \theta(k), \\
& c(k) \triangleq f^{\mathrm{T}}(k) R_{1}(k) f(k)+\operatorname{tr}\left[R_{3}(k) \theta^{\mathrm{T}}(k) R_{4}(k) \theta(k)\right] .
\end{aligned}
$$

Since $M(k)$ is positive definite, $J(\hat{\theta}, k)$ has the strict global minimizer $\theta(k+1)$ given by

$$
\theta(k+1)=-\frac{1}{2} \operatorname{vec}^{-1}\left[M^{-1}(k) b(k)\right],
$$


which requires the inverse of a positive-definite matrix of size $p l_{z} \times p l_{z}$. Equation (29) is the adaptive control update law. Note that $\bar{B}_{z u}$ (which appears in $f(k)$ and $D(k)$ ) must be specified in order to implement (29).

In the special case

$$
\begin{gathered}
R_{1}(k) \triangleq I_{p l_{z}}, \quad R_{12}(k) \triangleq 0_{p l_{z} \times l_{u}}, \quad R_{2}(k) \triangleq 0_{l_{u}}, \\
R_{3}(k) \triangleq \alpha(k) I_{n_{\mathrm{c}}\left(l_{u}+l_{y}\right)}, \quad R_{4}(k) \triangleq I_{l_{u}},
\end{gathered}
$$

where $\alpha(k)>0$ is a scalar, (26)-(28) become

$$
\begin{aligned}
M(k) & =D^{\mathrm{T}}(k) D(k)+\alpha(k) I, \\
b(k) & =2 D^{\mathrm{T}}(k) f(k)-2 \alpha(k) \operatorname{vec} \theta(k), \\
c(k) & =f^{\mathrm{T}}(k) f(k)+\alpha(k) \operatorname{tr}\left[\theta^{\mathrm{T}}(k) \theta(k)\right] .
\end{aligned}
$$

We use the weightings (30), (31) for all of the examples in this paper. The weighting parameter $\alpha(k)$ introduced in (31) is called the learning rate since it affects the convergence speed of the adaptive control algorithm. As $\alpha(k)$ is increased, a higher weight is placed on the difference between the previous controller coefficients and the updated controller coefficients, and, as a result, convergence speed is lowered. Likewise, as $\alpha(k)$ is decreased, convergence speed is raised. By varying $\alpha(k)$, we can effect tradeoffs between transient performance and convergence speed.

In the particular case $z=y$, using the retrospective performance variable $\hat{z}$ in place of $y$ in the regressor vector (13) yields faster convergence. Therefore, for $z=y$, we redefine (13) as

$$
\phi(k) \triangleq\left[\begin{array}{c}
\hat{z}(k-1) \\
\vdots \\
\hat{z}\left(k-n_{\mathrm{c}}\right) \\
u(k-1) \\
\vdots \\
u\left(k-n_{\mathrm{c}}\right)
\end{array}\right] .
$$

The novel feature of the adaptive control algorithm given by (11) and (29) is the use of the retrospective correction filter (RCF) (19), as shown in Figure 2 for $p=1$. RCF provides an inner loop to the adaptive control law by modifying the extended performance vector $Z(k)$ in terms of the difference between the actual past control inputs $U(k)$ and the recomputed control inputs $\hat{U}(\hat{\theta}, k)$, as given by (19).

\section{Markov-Parameter Polynomial}

By recursively substituting (1) into (3), it follows that $z(k)$ can be represented by

$$
\begin{aligned}
z(k)= & E_{1} A^{r} x(k-r)+H_{1} u(k-1)+H_{2} u(k-2)+\cdots+H_{r} u(k-r) \\
& +H_{z w, 0} w(k)+H_{z w, 1} w(k-1)+\cdots+H_{z w, r} w(k-r),
\end{aligned}
$$

where $r \geq d, H_{z w, 0} \triangleq E_{0}$, and, for all $i>0, H_{z w, i} \triangleq E_{1} A^{i-1} D_{1}$. In terms of the backward-shift operator $\mathbf{q}^{-1},(36)$ can be rewritten as

$$
\begin{aligned}
z(k)= & E_{1} A^{r} \mathbf{q}^{-r} x(k)+\left[H_{1} \mathbf{q}^{-1}+H_{2} \mathbf{q}^{-2}+\cdots+H_{r} \mathbf{q}^{-r}\right] u(k) \\
& +\left[H_{z w, 0}+H_{z w, 1} \mathbf{q}^{-1}+\cdots+H_{z w, r} \mathbf{q}^{-r}\right] w(k) .
\end{aligned}
$$

Shifting (37) forward by $r$ steps gives

$$
z(k+r)=E_{1} A^{r} x(k)+p_{r}(\mathbf{q}) u(k)+W_{r}(\mathbf{q}) w(k),
$$

where

$$
W_{r}(\mathbf{q}) \triangleq H_{z w, 0} \mathbf{q}^{r}+H_{z w, 1} \mathbf{q}^{r-1}+H_{z w, 2} \mathbf{q}^{r-2}+\cdots+H_{z w, r}
$$






Figure 2: Closed-loop system including adaptive control algorithm with the retrospective correction filter (dashed box) for $p=1$.

and

$$
p_{r}(\mathbf{q}) \triangleq H_{1} \mathbf{q}^{r-1}+H_{2} \mathbf{q}^{r-2}+\cdots+H_{r}
$$

We call $p_{r}(\mathbf{q})$ the Markov-parameter polynomial. Note that $p_{r}(\mathbf{q})$ is a matrix polynomial in the MIMO case and a polynomial in the SISO case. Furthermore, since $H_{1}=\cdots=H_{d-1}=0$ when $d \geq 2$, it follows that, for all $r \geq d \geq 1, p_{r}(\mathbf{q})$ can be written as

$$
p_{r}(\mathbf{q})=H_{d} \mathbf{q}^{r-d}+H_{d+1} \mathbf{q}^{r-d-1}+\cdots+H_{r} .
$$

The Markov-parameter polynomial $p_{r}(\mathbf{q})$ contains information about the relative degree $d$ and, in the SISO case, the sign of the high-frequency gain, that is, the sign of $H_{d}$. We show below that $p_{r}(\mathbf{q})$ also contains information about the transmission zeros of $G_{z u}(\mathbf{z}) \triangleq E_{1}(\mathbf{z} I-A)^{-1} B$, which is given by

$$
G_{z u}(\mathbf{z})=\frac{1}{\mathbf{z}^{n}+\alpha_{1} \mathbf{z}^{n-1}+\cdots+\alpha_{n}}\left(\beta_{1} \mathbf{z}^{n-1}+\beta_{2} \mathbf{z}^{n-2}+\cdots+\beta_{n}\right) .
$$

Recall that $\beta_{d}=H_{d}$.

In order to relate the transmission zeros of $G_{z u}$ to $p_{r}(\mathbf{q})$, the Laurent series expansion of $G_{z u}$ about $\mathbf{z}=\infty$ is given by

$$
G_{z u}(\mathbf{z})=\sum_{i=1}^{\infty} \mathbf{z}^{-i} H_{i} .
$$

This expansion converges uniformly on all compact subsets of $\{\mathbf{z}:|\mathbf{z}|>\rho(A)\}$, where $\rho(A)$ is the spectral radius of $A$ [25, Theorem 13, p. 186]. By truncating the summation in (43), we obtain the truncated Laurent 
expansion $\bar{G}_{r, z u}$ of $G_{z u}$, given by

$$
\bar{G}_{r, z u}(\mathbf{z}) \triangleq \sum_{i=1}^{r} \mathbf{z}^{-i} H_{i}=\frac{1}{\mathbf{z}^{r}}\left(H_{1} \mathbf{z}^{r-1}+\cdots+H_{r-1} \mathbf{z}+H_{r}\right)=\frac{1}{\mathbf{z}^{r}} p_{r}(\mathbf{z}) .
$$

Consequently, the Markov parameter matrix polynomial $p_{r}(\mathbf{q})$ is closely related to the truncated Laurent expansion of $G_{z u}$.

\section{V.A. Approximation of Outer Nonminimum-Phase Zeros}

In the case of MIMO systems, $p_{r}(\mathbf{q})$ is a matrix polynomial and thus does not have roots in the sense of a polynomial. We therefore require the notion of a Smith zero [?, p. 259]. Specifically, $\mathbf{z} \in \mathbb{C}$ is a $S m i t h$ zero of $p_{r}(\mathbf{q})$ if the rank of $p_{r}(\mathbf{z})$ is less than the normal rank of $p_{r}(\mathbf{q})$, that is, the maximum rank of $p_{r}(\xi)$ taken over all $\xi \in \mathbb{C}$. The notion of a Smith zero of a matrix polynomial is a direct consequence of the Smith decomposition.

Definition V.1. Let $\xi \in \mathbb{C}$ be a transmission zero of $G_{z u}$. Then, $\xi$ is an outer zero of $G_{z u}$ if $|\xi| \geq \rho(A)$. Otherwise, $\xi$ is an inner zero of $G_{z u}$.

The following result shows that the Smith zeros of the Markov parameter matrix polynomial $p_{r}(\mathbf{q})$ asymptotically approximate each outer transmission zero of $G_{z u}$.

Fact V.1. Let $\xi \in \mathbb{C}$ be an outer transmission zero of $G_{z u}$. For each $r$, let $\mathcal{R}_{r} \triangleq\left\{\xi_{r, 1}, \ldots, \xi_{r, m_{r}}\right\}$ denote the set of Smith zeros of $p_{r}(\mathbf{q})$. Then, there exists a sequence $\left\{\xi_{r, i_{r}}\right\}_{r=1}^{\infty}$ that converges to $\xi$ as $r \rightarrow \infty$.

The following specialization to SISO transfer functions shows that the roots of $p_{r}(\mathbf{q})$ asymptotically approximate each outer zero of $G_{z u}$.

Fact V.2. Consider $l_{u}=l_{z}=1$, and let $\xi \in \mathbb{C}$ be an outer zero of $G_{z u}$. For each $r$, let $\mathcal{R}_{r} \triangleq$ $\left\{\xi_{r, 1}, \ldots, \xi_{r, r-d}\right\}$ be the set of roots of $p_{r}(\mathbf{q})$. Then, there exists a sequence $\left\{\xi_{r, i_{r}}\right\}_{r=1}^{\infty}$ that converges to $\xi$ as $r \rightarrow \infty$.

The following examples illustrate Fact V.2 by showing that, as $r$ increases, roots of the Markov-parameter polynomial $p_{r}(\mathbf{q})$, and hence, roots of the numerator of the truncated transfer function $\bar{G}_{r, z u}$, asymptotically approximate each outer nonminimum-phase zero of $G_{z u}$. The remaining roots of $p_{r}(\mathbf{q})$ are either located at the origin or form an approximate ring with radius close to $\rho(A)$. These roots are spurious and have no effect on the adaptive control algorithm.

Example V.1 (SISO, nonminimum phase, stable plant). Consider the plant $G_{z u}$ with $d=2$, $H_{2}=1$, poles $0.5 \pm 0.5 \mathrm{\jmath},-0.5 \pm 0.5 \mathrm{\jmath}, \pm 0.95, \pm 0.7 \mathrm{\jmath}$, minimum-phase zeros $0.3 \pm 0.7 \mathrm{\jmath},-0.7 \pm 0.3 \mathrm{\jmath}$, and outer nonminimumphase zeros $1.25,-1.5$. Table 1 lists the approximated nonminimum-phase zeros obtained as roots of $p_{r}(\boldsymbol{q})$ as a function of $r$. Note that as $r$ increases, the outer nonminimum-phase zeros are more closely approximated by roots of $p_{r}(\boldsymbol{q})$. See Figure 3.

\begin{tabular}{|c||c|}
\hline$r$ & $\operatorname{roots}_{\mathrm{nmp}}\left(p_{r}(\mathbf{q})\right)$ \\
\hline \hline 6 & $\{\mathbf{0 . 9 4 4}, \mathbf{- 1 . 5 3 7}\}$ \\
\hline 8 & $\{\mathbf{1 . 1 7 0}, \mathbf{- 1 . 5 0 2}\}$ \\
\hline 10 & $\{\mathbf{1 . 2 0 7}, \mathbf{- 1 . 4 9 8}\}$ \\
\hline 15 & $\{\mathbf{1 . 2 4 0 , - 1 . 4 9 9}\}$ \\
\hline 20 & $\{\mathbf{1 . 2 4 8}, \mathbf{- 1 . 5 0 0}\}$ \\
\hline 25 & $\{\mathbf{1 . 2 5 0}, \mathbf{- 1 . 5 0 0}\}$ \\
\hline
\end{tabular}

Table 1: Approximated nonminimum-phase zeros obtained as roots of $p_{r}(\mathbf{q})$ as a function of $r$ for the stable, nonminimum-phase plant in Example V.1. As $r$ increases, the outer zeros are more accurately modeled. 


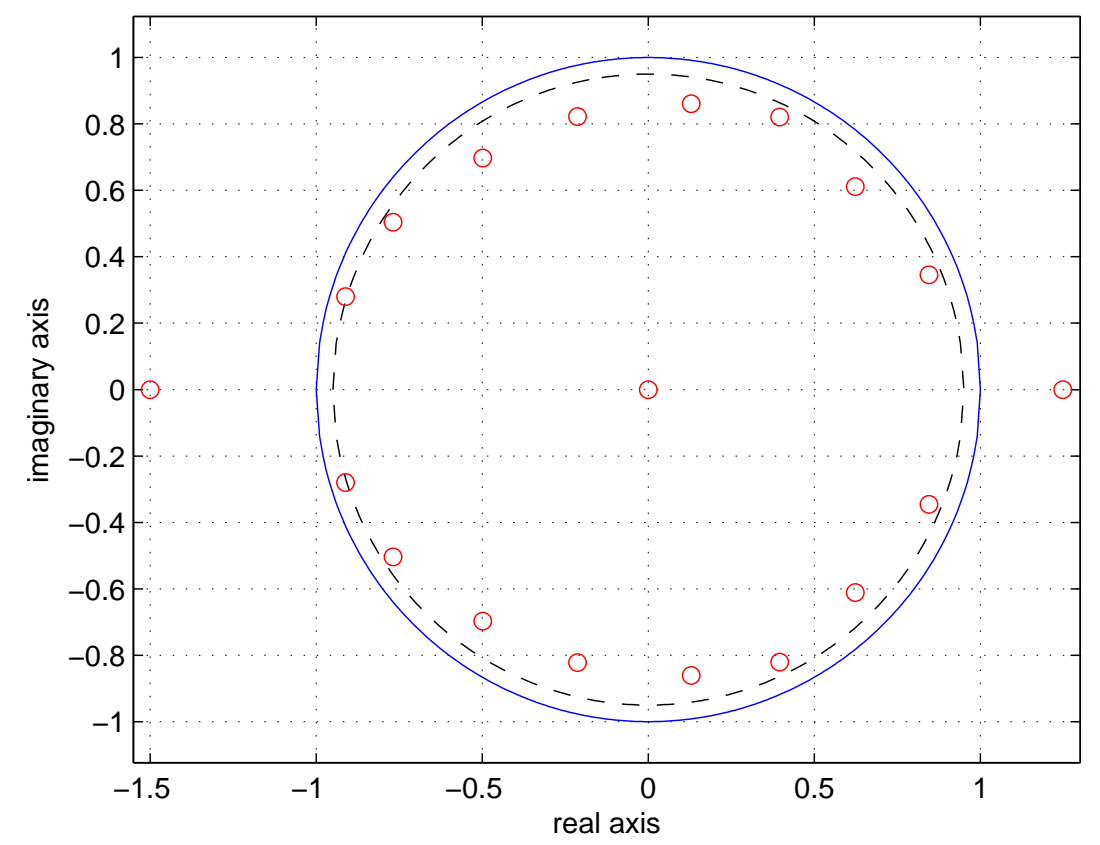

Figure 3: Roots of $p_{20}(\mathbf{q})$ for the stable, nonminimum-phase plant in Example V.1. The dashed line denotes $\rho(A)=$ 0.95. Note that the roots outside $\rho(A)$ are close to the outer nonminimum-phase zeros -1.5 and 1.25 . The remaining roots are either located at the origin or form an approximate ring with radius close to $\rho(A)$.

Example V.2 (SISO, nonminimum phase, unstable plant). Consider the plant $G_{z u}$ with $d=2, H_{2}=1$, poles $0.5 \pm 0.5 \mathrm{\jmath},-0.5 \pm 0.5 \mathrm{\jmath}, \pm 0.7 \mathrm{\jmath},-0.95,1.4$, minimum-phase zeros $0.3 \pm 0.7 \mathrm{\jmath},-0.7 \pm 0.3 \mathrm{\jmath}$, outer nonminimumphase zero -1.5, and inner nonminimum-phase zero 1.25. Figure 4 shows the roots of $p_{25}(\boldsymbol{q})$. Note that the root of $p_{25}(\boldsymbol{q})$ outside $\rho(A)$ is close to the outer nonminimum-phase zero -1.5. However, the inner nonminimum-phase zero 1.25 is not approximated by a root of $p_{25}(\boldsymbol{q})$. The remaining roots are either located at the origin or form an approximate ring with radius close to $\rho(A)$.

\section{V.B. Approximation of Inner Nonminimum-Phase Zeros}

Example V.2 illustrates that the roots of $p_{r}(\mathbf{q})$ approximate each outer nonminimum-phase zero of $G_{z u}$. However, inner nonminimum-phase zeros of $G_{z u}$ are not approximated by roots of $p_{r}(\mathbf{q})$. To overcome this deficiency, we can use information about the plant's unstable poles to create a modified Markov-parameter polynomial $\tilde{p}_{r}(\mathbf{q})$ whose roots approximate each nonminimum-phase zero of $G_{z u}$. For illustration, assume that the SISO plant $G_{z u}$ has a unique unstable pole $\zeta \in \mathbb{C}$ whose absolute value is greater than all other poles of $G_{z u}$. Then, we define

$$
\begin{aligned}
\tilde{G}_{z u}(\mathbf{z}) & \triangleq \frac{\mathbf{z}-\zeta}{\mathbf{z}} G_{z u}(\mathbf{z})=G_{z u}(\mathbf{z})-\frac{\zeta}{\mathbf{z}} G_{z u}(\mathbf{z}) \\
& =\sum_{i=d}^{\infty} \mathbf{z}^{-i} H_{i}-\sum_{i=d}^{\infty} \mathbf{z}^{-(i+1)} \zeta H_{i}=\sum_{i=d}^{\infty} \mathbf{z}^{-i}\left[H_{i}-\zeta H_{i-1}\right] \\
& =\sum_{i=d}^{\infty} \mathbf{z}^{-i} \tilde{H}_{i},
\end{aligned}
$$

where, for $i=1,2, \ldots, \tilde{H}_{i} \triangleq H_{i}-\zeta H_{i-1}$ are the modified Markov parameters, and $H_{0}=0$. By repeating this operation for each unstable pole of $G_{z u}$, the roots of the modified Markov-parameter polynomial

$$
\tilde{p}_{r}(\mathbf{q}) \triangleq \tilde{H}_{d} \mathbf{q}^{r-d}+\tilde{H}_{d+1} \mathbf{q}^{r-d-1}+\cdots+\tilde{H}_{r}
$$

can approximate each nonminimum-phase zero of $G_{z u}$. The following example illustrates this process. 


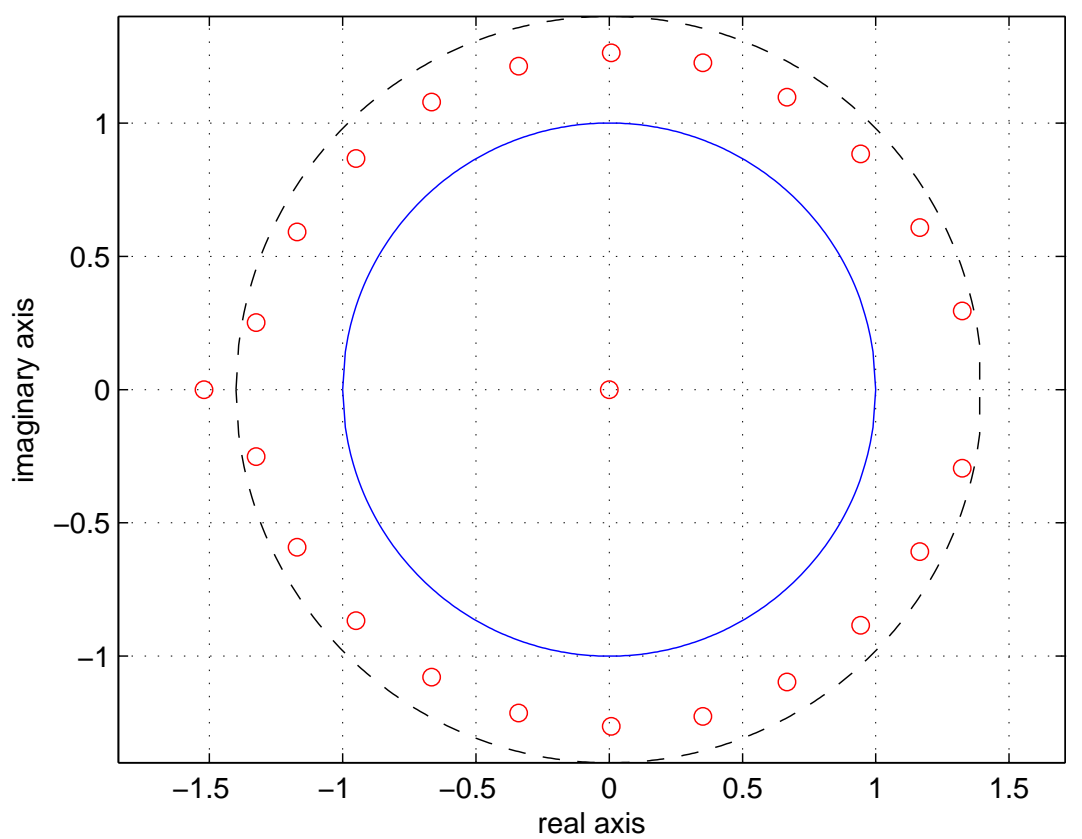

Figure 4: Roots of $p_{25}(\mathbf{q})$ for the unstable, nonminimum-phase plant in Example V.2. The dashed line denotes $\rho(A)=1.4$. Note that the root of $p_{25}(\mathbf{q})$ outside $\rho(A)$ is close to the outer nonminimum-phase zero -1.5 . However, the nonminimum-phase zero 1.25 is not approximated by a root of $p_{25}(\mathbf{q})$. The remaining roots are either located at the origin or form an approximate ring with radius close to $\rho(A)$.

Example V.3 (Ex. V.2 with pole information). Reconsider Example V.2, where the inner nonminimumphase zero 1.25 is not approximated by a root of $p_{r}(\boldsymbol{q})$. Using knowledge of the unstable pole 1.4 to construct $\tilde{p}_{r}(\boldsymbol{q})$ given by (46), Figure 5 shows the roots of $\tilde{p}_{25}(\boldsymbol{q})$. Note that the roots outside $\rho(\tilde{A})$, where $\tilde{A}$ is the dynamics matrix of a minimal realization of $\tilde{G}_{z u}$, are close to the nonminimum-phase zeros of $G_{z u}$. The remaining roots are either located at the origin or form an approximate ring with radius close to $\rho(\tilde{A})$.

\section{Construction of $\bar{B}_{z u}$}

We present four constructions for $\bar{B}_{z u}$ based on the available modeling information.

\section{VI.A. $B_{z u}$-Based Construction}

If $B_{z u}$ given by (8) is known, then, with $r=1, \bar{B}_{z u}$ can be chosen to be equal to $B_{z u}$. In this case, (17) becomes

$$
\hat{Z}(\hat{\theta}, k)=W_{z w} \phi_{z w}(k)+B_{z u} \hat{U}(\hat{\theta}, k) .
$$

This construction of $\bar{B}_{z u}$ captures information about the relative degree $d$, the sign of the high-frequency gain, and exact values of all transmission zeros of $G_{z u}$, that is, both minimum-phase and nonminimum-phase transmission zeros.

\section{VI.B. Nonminimum-Phase-Zero-Based Construction}

Consider $l_{u}=l_{z}=1, r=1$, and assume that $H_{d}$ and the nonminimum-phase zeros of $G_{z u}$ are known. Then we define the nonminimum-phase numerator polynomial $N(\mathbf{q})$ to be the polynomial whose roots are equal to the nonminimum-phase zeros of $G_{z u}$, that is,

$$
N(\mathbf{q}) \triangleq H_{d} \mathbf{q}^{m}+\tilde{\beta}_{1} \mathbf{q}^{m-1}+\cdots+\tilde{\beta}_{m},
$$




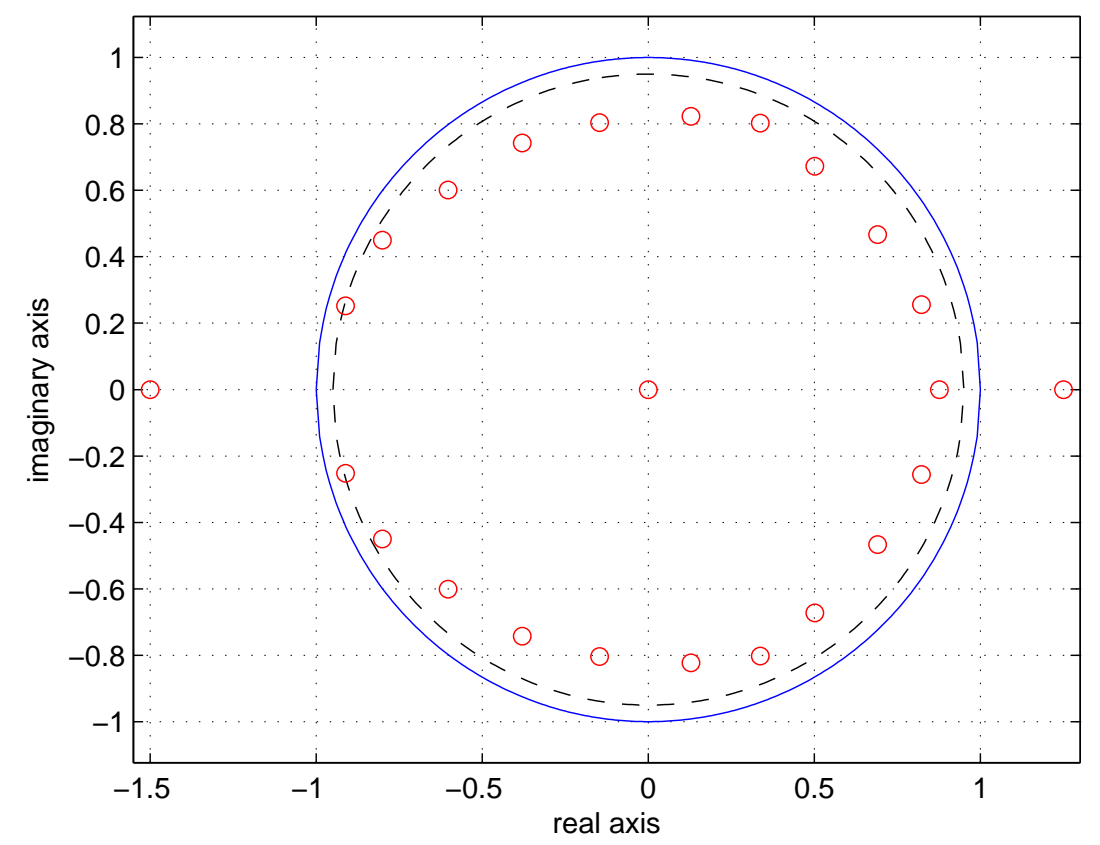

Figure 5: Roots of $\tilde{p}_{25}(\mathbf{q})$ for the unstable, nonminimum-phase plant in Example V.3. The dashed line denotes $\rho(\tilde{A})=0.95$, where $\tilde{A}$ is the dynamics matrix of a minimal realization of $\tilde{G}_{z u}$. Note that the roots outside $\rho(\tilde{A})$ are close to the inner and outer nonminimum-phase zeros of $G_{z u}$. The remaining roots are either located at the origin or form an approximate ring with radius close to $\rho(\tilde{A})$.

where $m \geq 0$ is the number of nonminimum-phase zeros in $G_{z u}$, and $\tilde{\beta}_{1}, \ldots, \tilde{\beta}_{m} \in \mathbb{R}$. If $m=0$, that is, $G_{z u}$ is minimum phase, then $N(\mathbf{q})=H_{d}$. The nonminimum-phase-zero-based construction of $\bar{B}_{z u}$ is thus given by

$$
\bar{B}_{z u}=\left[\begin{array}{cccccccccccc}
H_{1} & \cdots & H_{d} & \tilde{\beta}_{1} & \cdots & \tilde{\beta}_{m} & 0_{l_{z} \times l_{u}} & \cdots & 0_{l_{z} \times l_{u}} & 0_{l_{z} \times l_{u}} & \cdots & 0_{l_{z} \times l_{u}} \\
0_{l_{z} \times l_{u}} & \ddots & & \ddots & \ddots & & \ddots & \ddots & & \ddots & \ddots & \vdots \\
\vdots & \ddots & \ddots & & \ddots & \ddots & & \ddots & \ddots & & \ddots & \vdots \\
0_{l_{z} \times l_{u}} & \cdots & 0_{l_{z} \times l_{u}} & H_{1} & \cdots & H_{d} & \tilde{\beta}_{1} & \cdots & \tilde{\beta}_{m} & 0_{l_{z} \times l_{u}} & \cdots & 0_{l_{z} \times l_{u}}
\end{array}\right] .
$$

This construction of $\bar{B}_{z u}$ captures information about the relative degree $d$, the sign of the high-frequency gain, and exact values of all nonminimum-phase zeros of $G_{z u}$. Note that, in the minimum-phase case, the only required modeling information is $H_{d}$. A MIMO extension of this construction, utilizing the Smith-McMillan form of $G_{z u}$, is presented in. ${ }^{26}$

\section{VI.C. $r$-MARKOV-Based Construction}

Replacing $k$ with $k-1$ in (4) and substituting the resulting relation back into (4) yields a 2-MARKOV model. Repeating this procedure $r-1$ times yields the $r$-MARKOV model of (1)-(3)

$$
\begin{aligned}
z(k)= & \sum_{i=1}^{n} \alpha_{r, i} z(k-r-i+1)+\sum_{i=d}^{r-1} H_{i} u(k-i)+\sum_{i=1}^{n} \beta_{r, i} u(k-r-i+1) \\
& +\sum_{i=0}^{r-1} H_{z w, i} w(k-i)+\sum_{i=1}^{n} \gamma_{r, i} w(k-r-i+1),
\end{aligned}
$$


where, for $i=1, \ldots, n$, the coefficients $\alpha_{r, i} \in \mathbb{R}, \beta_{r, i} \in \mathbb{R}^{l_{z} \times l_{u}}$, and $\gamma_{r, i} \in \mathbb{R}^{l_{z} \times l_{w}}$ are given by

$$
\begin{array}{rlrl}
\alpha_{1, i} \triangleq-\alpha_{i}, & \beta_{1, i} \triangleq \beta_{i}, & \gamma_{1, i} \triangleq \gamma_{i}, \\
& \vdots & \vdots & \vdots \\
\alpha_{r, i} \triangleq \alpha_{r-1,1} \alpha_{1, i}+\alpha_{r-1, i+1}, & \beta_{r, i} \triangleq \alpha_{r-1,1} \beta_{1, i}+\beta_{r-1, i+1}, & \gamma_{r, i} \triangleq \alpha_{r-1,1} \gamma_{1, i}+\gamma_{r-1, i+1}, \\
& \vdots & \vdots & \vdots \\
\alpha_{r, n} \triangleq \alpha_{r-1,1} \alpha_{1, n}, & \beta_{r, n} \triangleq \alpha_{r-1,1} \beta_{1, n}, & \gamma_{r, n} \triangleq \alpha_{r-1,1} \gamma_{1, n} .
\end{array}
$$

Note that $\beta_{r, 1}=H_{r}$ and $\gamma_{r, 1}=H_{z w, r}$. We represent (50) with $w=0$ as the $r$-MARKOV transfer function

$$
\begin{aligned}
G_{r, z u}(\mathbf{z})= & \frac{1}{\mathbf{z}^{r+n-1}+\alpha_{r, 1} \mathbf{z}^{n-1}+\cdots+\alpha_{r, n}} \\
& \cdot\left(H_{1} \mathbf{z}^{r+n-2}+\cdots+H_{r-1} \mathbf{z}^{n}+H_{r} \mathbf{z}^{n-1}+\beta_{r, 2} \mathbf{z}^{n-2}+\cdots+\beta_{r, n}\right) .
\end{aligned}
$$

The system representation (52) is nonminimal since its order is $n+r-1$, and thus (52) includes poles that are not present in the original model. Furthermore, note that the coefficients of the terms $\mathbf{z}^{n+r-2}$ through $\mathbf{z}^{n}$ in the denominator are zero. These facts are irrelevant for the following development. Using the numerator coefficients of (52), the $r$-MARKOV-based construction of $\bar{B}_{z u}$ is given by

$$
\bar{B}_{z u}=\left[\begin{array}{ccccccccc}
H_{1} & \cdots & H_{r} & \beta_{r, 2} & \cdots & \beta_{r, n} & 0_{l_{z} \times l_{u}} & \cdots & 0_{l_{z} \times l_{u}} \\
0_{l_{z} \times l_{u}} & \ddots & & \ddots & \ddots & & \ddots & \ddots & \vdots \\
\vdots & \ddots & \ddots & & \ddots & \ddots & & \ddots & 0_{l_{z} \times l_{u}} \\
0_{l_{z} \times l_{u}} & \cdots & 0_{l_{z} \times l_{u}} & H_{1} & \cdots & H_{r} & \beta_{r, 2} & \cdots & \beta_{r, n}
\end{array}\right] .
$$

This construction of $\bar{B}_{z u}$ captures information about the relative degree $d$, the sign of the high-frequency gain, and exact values of all transmission zeros of $G_{z u}$, that is, both minimum-phase and nonminimum-phase transmission zeros.

\section{VI.D. Markov-Parameter-Based Construction}

Using the numerator coefficients of (44), the Markov-parameter-based construction of $\bar{B}_{z u}$ is given by

$$
\bar{B}_{z u}=\left[\begin{array}{ccccccccc}
H_{1} & \cdots & H_{r} & 0_{l_{z} \times l_{u}} & \cdots & 0_{l_{z} \times l_{u}} & 0_{l_{z} \times l_{u}} & \cdots & 0_{l_{z} \times l_{u}} \\
0_{l_{z} \times l_{u}} & \ddots & & \ddots & \ddots & & \ddots & \ddots & \vdots \\
\vdots & \ddots & \ddots & & \ddots & \ddots & & \ddots & \vdots \\
0_{l_{z} \times l_{u}} & \cdots & 0_{l_{z} \times l_{u}} & H_{1} & \cdots & H_{r} & 0_{l_{z} \times l_{u}} & \cdots & 0_{l_{z} \times l_{u}}
\end{array}\right] .
$$

The Markov parameters are the numerator coefficients of a truncated Laurent series expansion of $G_{z u}$ about $\mathbf{z}=\infty$. The Markov parameters contain information about the relative degree $d$, the sign of the highfrequency gain, and, as shown by Fact V.2 for the SISO case, approximate values of all outer nonminimumphase zeros of $G_{z u}$. The advantage in using $\bar{B}_{z u}$ given by (54) rather than (53) is that $\beta_{r, 2}, \ldots, \beta_{r, n}$ need not be known. If, however, $G_{z u}$ has inner nonminimum-phase zeros and the unstable poles of $G_{z u}$ whose absolute values are greater than at least one inner nonminimum-phase zero are known, then we replace the Markov parameters $H_{1}, \ldots, H_{r}$ in (54) by the modified Markov parameters $\tilde{H}_{1}, \ldots, \tilde{H}_{r}$ given in (45). If these poles are not known, then $\bar{B}_{z u}$ can be chosen to be $B_{z u}$, the nonminimum-phase zero form given by (49), or the $r$-MARKOV form given by (53). 


\section{Adaptive Control of GTM}

We now apply RCF adaptive control to the Generic Transport Model (GTM), a twin-engine transportclass nonlinear aircraft simulation developed at NASA Langley Research Center for aviation safety research. Specifically, we use RCF control for disturbance rejection and command-following under nominal and failure conditions. All of the examples are based around a nominal trim condition with the following parameters:

1. Flight path angle of $0.00 \mathrm{deg}$.

2. Body $x-, y-$, and $z$-axis velocities of $161.66,0.00$, and $7.12 \mathrm{ft} / \mathrm{s}$.

3. Angular velocities in roll, pitch, and yaw of 0.00, 0.00, and 0.00 degrees/s.

4. Latitude, longitude, and altitude of 37.03 degrees, -76.5 degrees, and $625.48 \mathrm{ft}$.

5. Roll, pitch, and yaw angles of $0.077,2.52$, and 90 degrees.

Additionally, for all of the examples below we choose the performance variable vector

$$
z=\left[\begin{array}{c}
\Phi \\
r \\
\left(h-h_{\mathrm{ref}}\right) / 30
\end{array}\right],
$$

where the roll angle $\Phi$, body yaw rate $r$, and altitude $h$ are in $\mathrm{rad}, \mathrm{rad} / \mathrm{s}$, and $\mathrm{ft}$, respectively. Note that the altitude is scaled by 30 so that its magnitude is comparable to the remaining performance variables. Here $h_{\text {ref }}$ represents the desired altitude. We do not assume that air speed is measured, and we will use the Markov-parameter-based construction of $\bar{B}_{z u}$, given by (54).

Example VII.1 (Nominal Disturbance Rejection). We begin with a disturbance rejection example in which there is a periodic downward wind gust of $0.2 \mathrm{ft} / \mathrm{s}$ with a period of $100 \mathrm{sec}$ as shown in Figure 6 . As a basis

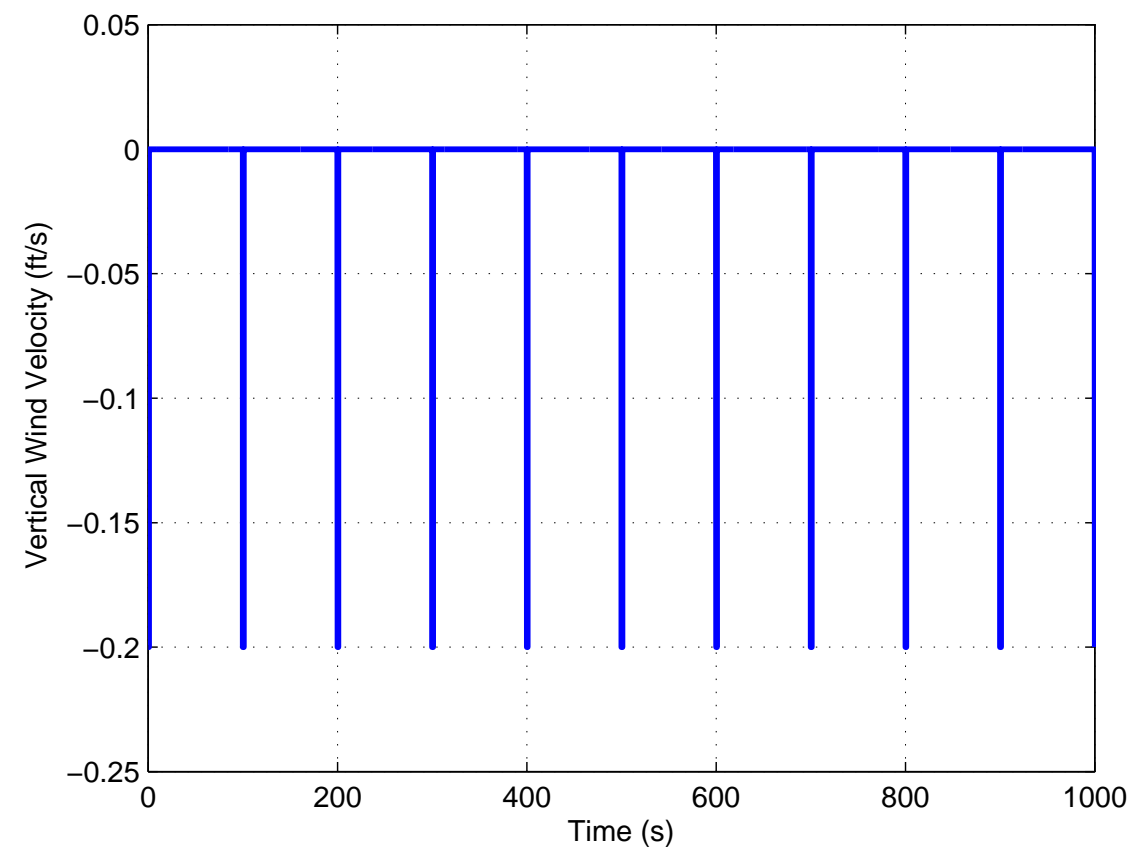

Figure 6: Downward wind gusts.

of comparison, the nominal aircraft response is shown in Figure 7, where it can be seen that the aircraft altitude does not stabilize at the nominal trim condition. Next, we apply RCF adaptive control to the aircraft to stabilize the aircraft at its nominal trim condition. For the adaptive control law we let the measurement vector be identical to the performance variable vector $y=z$, and choose $r=5, n_{c}=1, p=1$, and $\alpha=10$. As 
inputs we choose the rudder, elevator, and ailerons. The nominal linearized model between control surfaces and performance variables is provided by the GTM simulation. Figure 8 shows the aircraft response when RCF adaptive control is applied. It can be seen that the altitude is stabilized and approaches the nominal trim condition. Figure 9 shows the control surface deflections due to the control law.

Example VII.2 (Disturbance Rejection with Elevator Failure). Consider again disturbance rejection of the periodic wind gust as shown in Figure 6, where we now introduce an effectiveness failure in the elevator. That is, the elevator commands are now taken to be $75 \%$ less effective than the nominal model dictates, although this change is not modeled by $B_{z u}$. We consider the same controller parameters as in the failure-free case. Figure 10 shows that, despite the failure, the altitude is again stabilized and all of the performance variables approach their trim values. Figure 11 shows the control surface deflections due to the control law.

Example VII.3 (Nominal Command Following). Next, we consider command following of the altitude doublet shown in Figure 12, in the presence of zero-mean Gaussian North, East, and Vertical winds with covariances of $0.0001 \mathrm{ft} / \mathrm{s}$. For this case, $h_{\text {ref }}$ represents the commanded altitude. We also augment the measurement vector to include the doublet command, that is,

$$
y=\left[\begin{array}{c}
\Phi \\
r \\
\left(h-h_{\mathrm{ref}}\right) / 30 \\
h_{\mathrm{ref}} / 30
\end{array}\right] .
$$

Note that since $y$ now includes the command, the adaptive control law contains both feedback and feedforward components. We choose the controller parameters $r=5, n_{c}=1, p=1$, and $\alpha=10$, as in the previous examples. Figure 13 shows the aircraft response to the altitude doublet command under RCF adaptive control. Figure 14 shows the control surface deflections due to the control law.

Example VII.4 (Command Following with Elevator Failure). We again consider command following of the altitude doublet as shown in Figure 12 in the presence of Gaussian North, East, and Vertical winds, where we introduce an effectiveness failure in the elevator, now taken to be $75 \%$ less effective than the nominal model dictates. We consider the same controller parameters as in the failure-free case. Figure 15 shows that, despite the failure, the performance variable vector is able to follow the altitude doublet. Figure 16 shows the control surface deflections due to the control law.

\section{Conclusion}

We presented a detailed description of retrospective-cost based adaptive control, which is a discrete-time adaptive control law capable of stabilization, command following, and disturbance rejection for systems that are unstable, MIMO, and/or nonminimum phase. We then illustrated the effectiveness of the RCF algorithm for disturbance rejection and command following on NASA's Generic Transport Model both nominally and under unknown, reduced controller authority.

\section{Acknowledgments}

This research has been supported in part by the DoD under a National Defense Science and Engineering Graduate Fellowship, and NASA under IRAC Grant NNX08AB92A, Dr. S. Joshi, contract monitor.

\section{References}

${ }^{1}$ Narendra, K. S. and Annaswamy, A. M., Stable Adaptive Systems, Prentice Hall, Englewood Cliffs, New Jersey, 1989.

${ }^{2}$ Goodwin, G. C., Ramadge, P. J., and Caines, P. E., "Discrete-time multivariable adaptive control," IEEE Trans. Autom. Contr., Vol. 25, 1980, pp. 449-456.

${ }^{3}$ Hoagg, J. B., Santillo, M. A., and Bernstein, D. S., "Discrete-Time Adaptive Command Following and Disturbance Rejection with Unknown Exogenous Dynamics," IEEE Trans. Autom. Contr., Vol. 53, 2008, pp. 912-928.

${ }^{4}$ Åström, K. J., Hagander, P., and Sternby, J., "Zeros of Sampled Systems," Automatica, Vol. 20, 1984, pp. 31-38.

${ }^{5}$ Bai, E. and Sastry, S., "Persistency of excitation, sufficient richness and parameter convergence in discrete-time adaptive control," Sys. Contr. Lett., Vol. 6, 1985, pp. 153-163. 
${ }^{6}$ Rohrs, C., Valavani, L., Athans, M., and Stein, G., "Robustness of continuous-time adaptive control algorithms in the presence of unmodeled dynamics," IEEE TAC, Vol. 30, 1985, pp. 881-889.

${ }^{7}$ Ioannou, P. and Sun, J., Robust Adaptive Control, Prentice Hall, 1996.

${ }^{8}$ Ohkawa, F. and Yonezawa, Y., "A discrete model reference adaptive control system for a plant with input amplitude constraints," Int. J. Contr., Vol. 36, 1982, pp. 747-753.

${ }^{9}$ Zhang, C. and Evans, R., "Amplitude constrained adaptive control," Int. J. Contr., Vol. 46, 1987, pp. 53-64.

${ }^{10}$ Karason, S. and Annaswamy, A., "Adaptive control in the presence of input constraints," IEEE TAC, Vol. 39, 1994, pp. 2325-2330.

${ }^{11}$ Lai, W. and Cook, P., "A discrete-time universal regulator," Int. J. Contr., Vol. 62, 1995, pp. 17-32.

${ }^{12}$ Lindquist, A. and Yakubovich, V., "Universal regulators for optimal tracking in discrete-time systems affected by harmonic disturbances," IEEE Trans. Autom. Contr., Vol. 44, 1999, pp. 1688-1704.

${ }^{13}$ Anderson, B., "Topical Problems of Adaptive Control," Proc. European Contr. Conf., Kos, Greece, July 2007, pp. 4997-4998.

${ }^{14}$ Goodwin, G. C. and Sin, K. S., Adaptive Filtering, Prediction, and Control, Prentice Hall, 1984.

${ }^{15}$ Johansson, R., "Global Lyapunov stability and exponential convergence of direct adaptive control," Int. J. Contr., Vol. 50, 1989, pp. 859-869.

${ }^{16}$ Mareels, I. and Polderman, J. W., Adaptive Systems: An Introduction, Birkhäuser, Boston, MA, 1996.

${ }^{17}$ Hayakawa, T., Haddad, W. M., and Leonessa, A., "A Lyapunov-based adaptive control framework for discrete-time non-linear systems with exogenous disturbances," Int. J. Contr., Vol. 77, 2004, pp. 250-263.

${ }^{18}$ Akhtar, S. and Bernstein, D. S., "Lyapunov-stable discrete-time model reference adaptive control," Int. J. Adaptive Contr. Signal Proc., Vol. 19, 2005, pp. 745-767.

${ }^{19}$ Bayard, D. S., "Extended horizon liftings for stable inversion of nonminimum-phase systems," IEEE Trans. Autom. Contr., Vol. 39, 1994, pp. 1333-1338.

${ }^{20}$ Venugopal, R. and Bernstein, D. S., "Adaptive disturbance rejection using ARMARKOV/Toeplitz Models," IEEE Trans. Contr. Sys. Tech., Vol. 8, 2000, pp. 257-269.

${ }^{21}$ Hoagg, J. B., Santillo, M. A., and Bernstein, D. S., "Internal Model Control in the Shift and Delta Domains," IEEE Trans. Autom. Contr., Vol. 53, 2008, pp. 1066-1072.

${ }^{22}$ Bailey, R., Hostetler, R., Barnes, K., Belcastro, C., and Belcastro, C., "Experimental Validation: Subscale Aircraft Ground Facilities and Integrated Test Capability," Proc. Guid. Nav. Contr. Conf., San Francisco, CA, August 2005, AIAA2005-6433.

${ }^{23}$ Jordan, T. and Bailey, R., "NASA Langley's AirSTAR Testbed: A Subscale Flight Test Capability for Flight Dynamics and Control System Experiments," Proc. Guid. Nav. Contr. Conf., Honolulu, HI, August 2008, AIAA-2008-6660.

${ }^{24}$ Murch, A., "A Flight Control System Architecture for the NASA AirSTAR Flight Test Infrastructure," Proc. Guid. Nav. Contr. Conf., Honolulu, HI, August 2008, AIAA-2008-6990.

${ }^{25}$ Marsden, J. E., Basic Complex Analysis, W. H. Freeman, 1973.

${ }^{26}$ Santillo, M. A. and Bernstein, D. S., "A Retrospective Correction Filter for Discrete-time Adaptive Control of Nonminimum Phase Systems," Proc. Conf. Dec. Contr., Cancun, Mexico, December 2008, pp. 690-695. 


\section{Performance Variables}
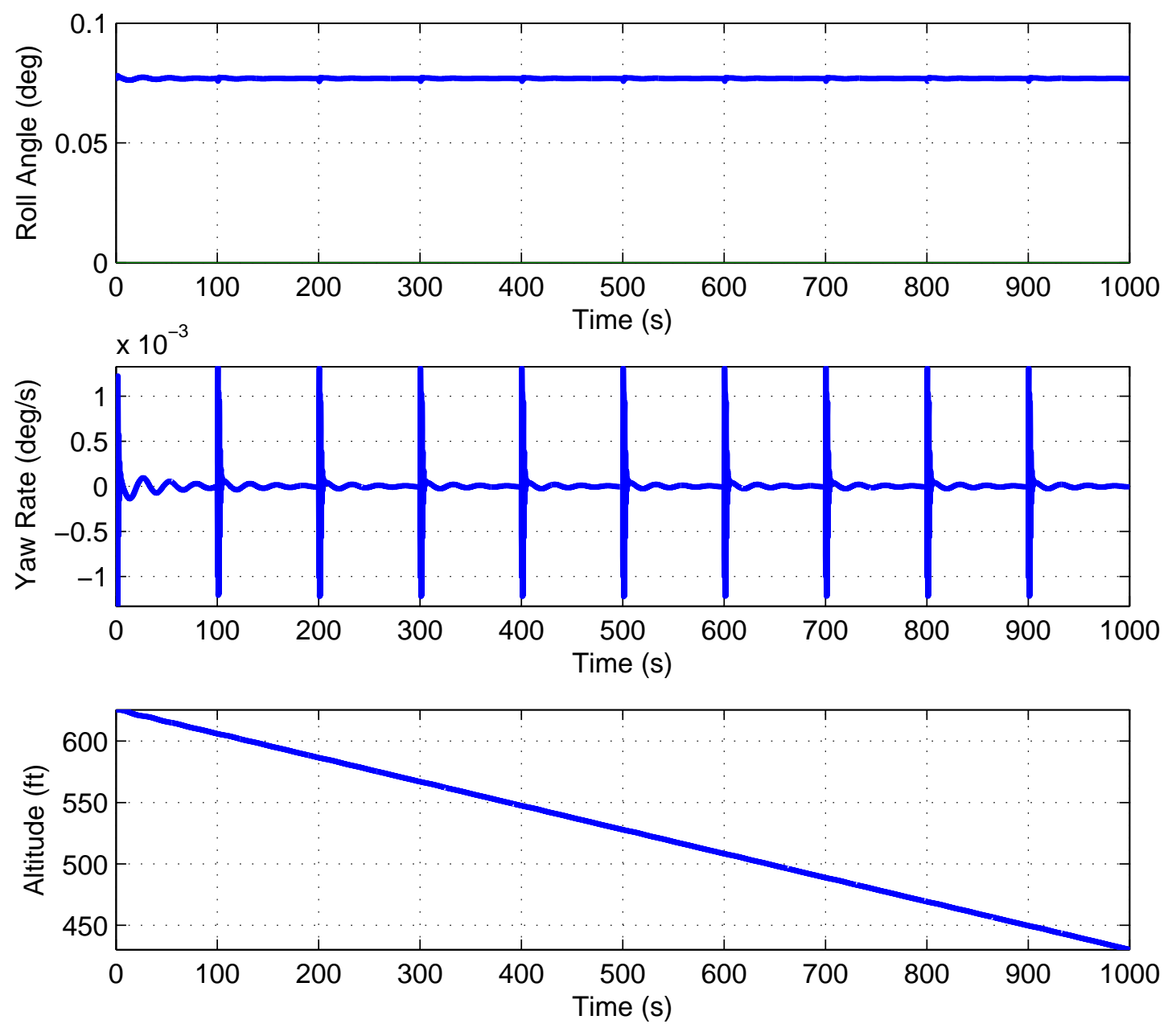

Figure 7: Nominal aircraft response to downward wind gusts shown in Figure 6. 


\section{Performance Variables}
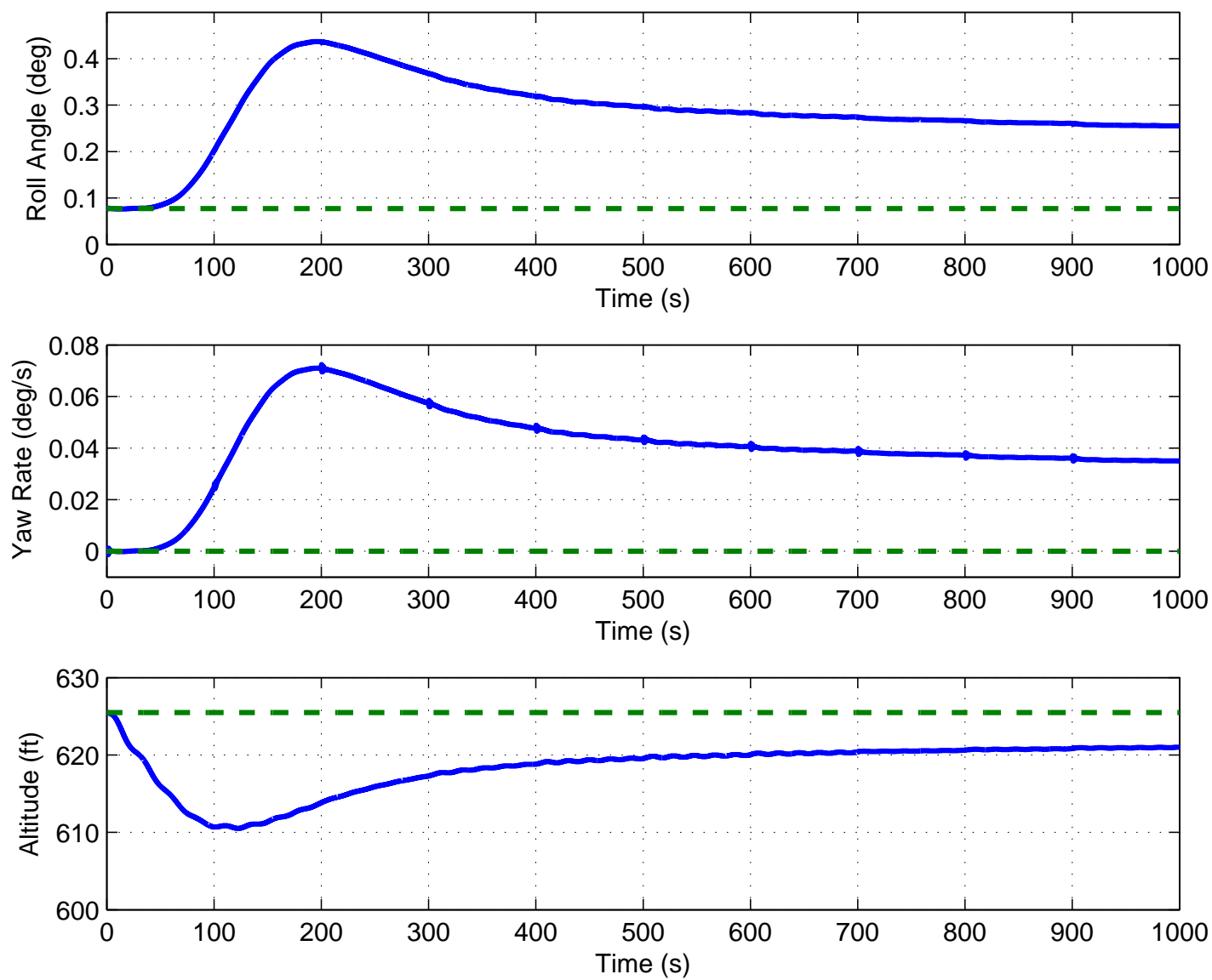

Figure 8: Aircraft response to periodic downward wind gusts shown in Figure 6 under RCF adaptive control. The solid lines represent true aircraft response, while the dashed lines represent desired performance. 


\section{Control Surfaces}
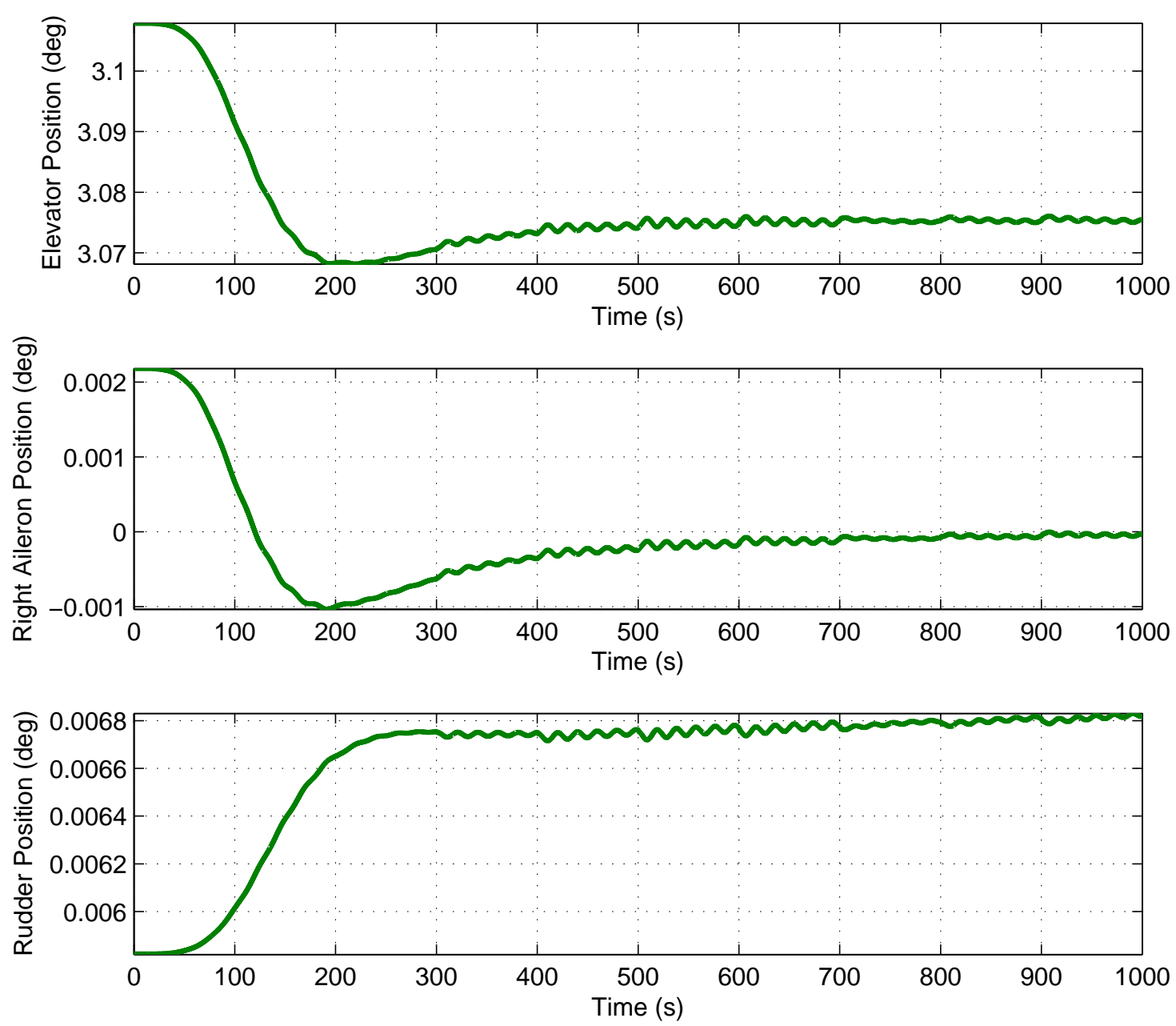

Figure 9: Aircraft control surface deflections due to the RCF adaptive control in response to the downward wind gusts shown in Figure 6. 


\section{Performance Variables}
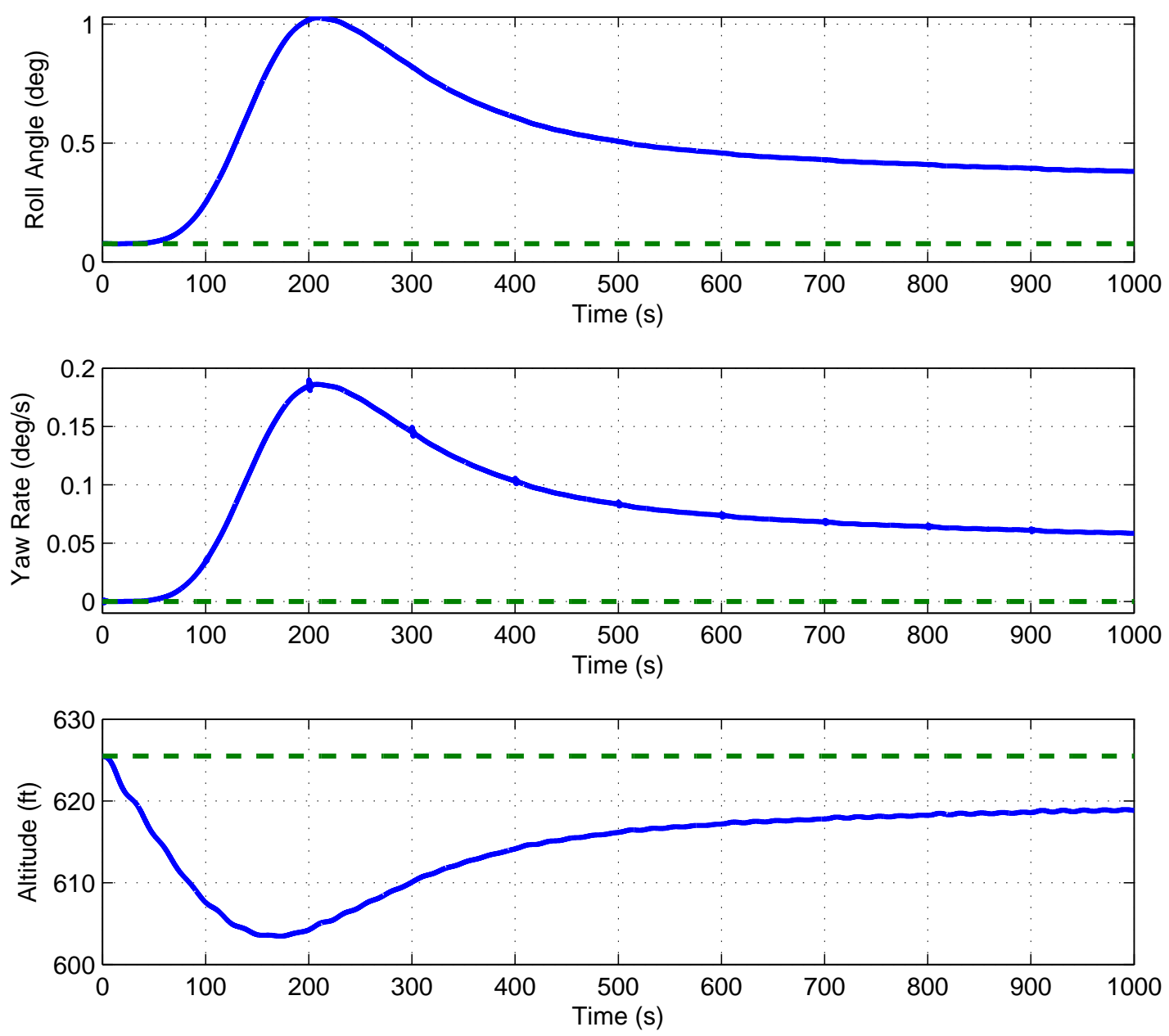

Figure 10: Aircraft response under RCF adaptive control to downward wind gusts shown in Figure 6 with a $75 \%$ reduction in elevator effectiveness. The solid lines represent true aircraft response, while the dotted lines represent desired performance. 


\section{Control Surfaces}
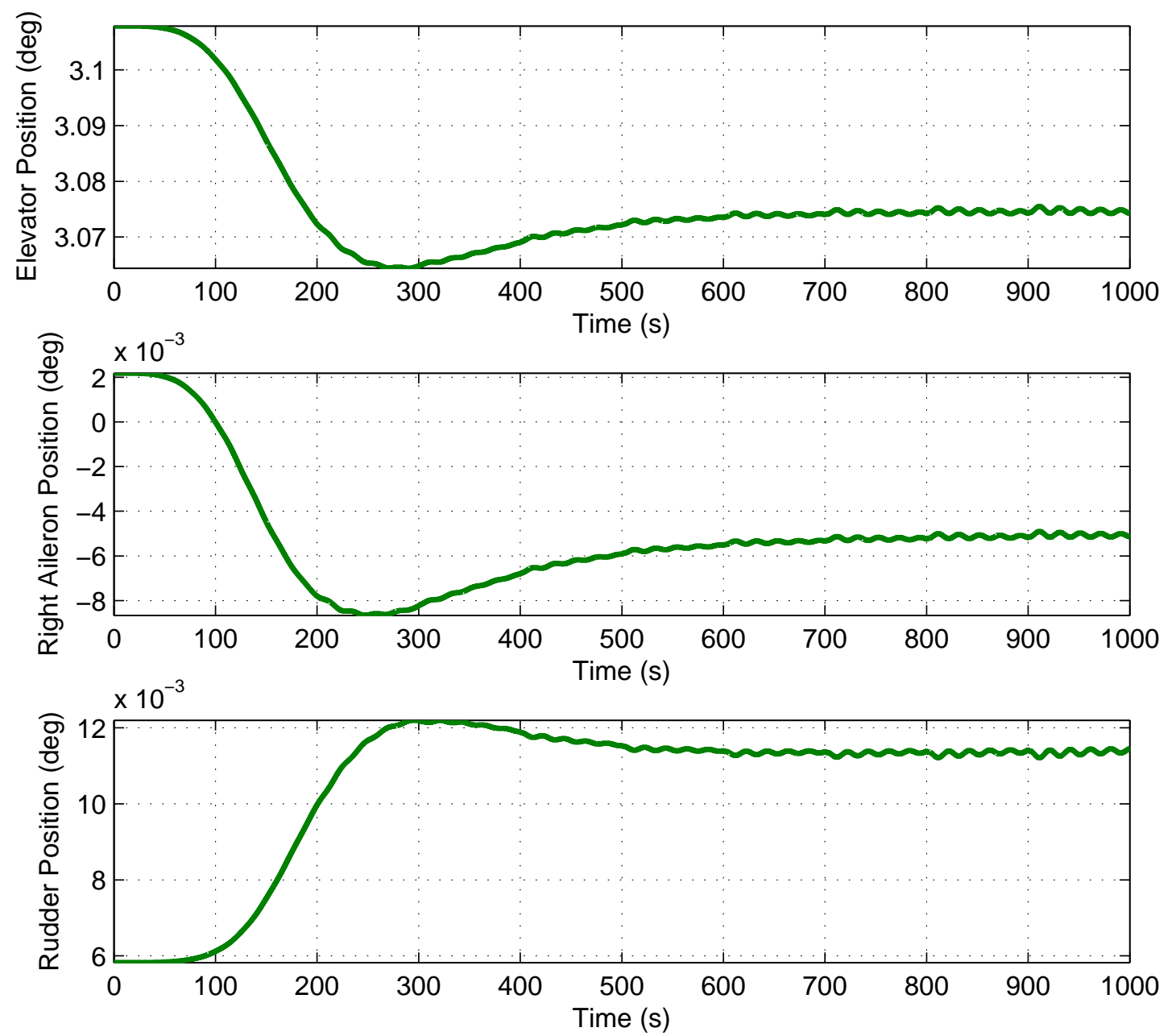

Figure 11: Aircraft control surface deflections due to RCF adaptive control in response to the downward wind gusts shown in Figure 6 with a $75 \%$ reduction in elevator effectiveness. 


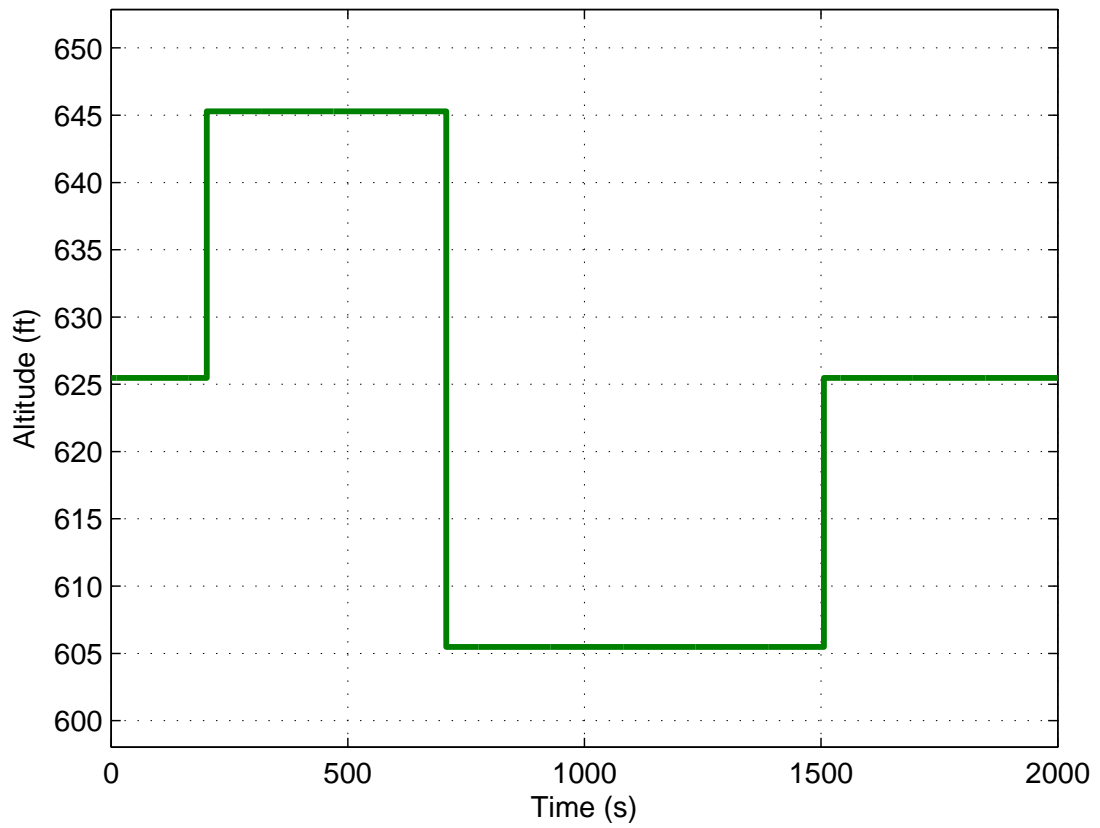

Figure 12: Commanded altitude doublet. 


\section{Performance Variables}
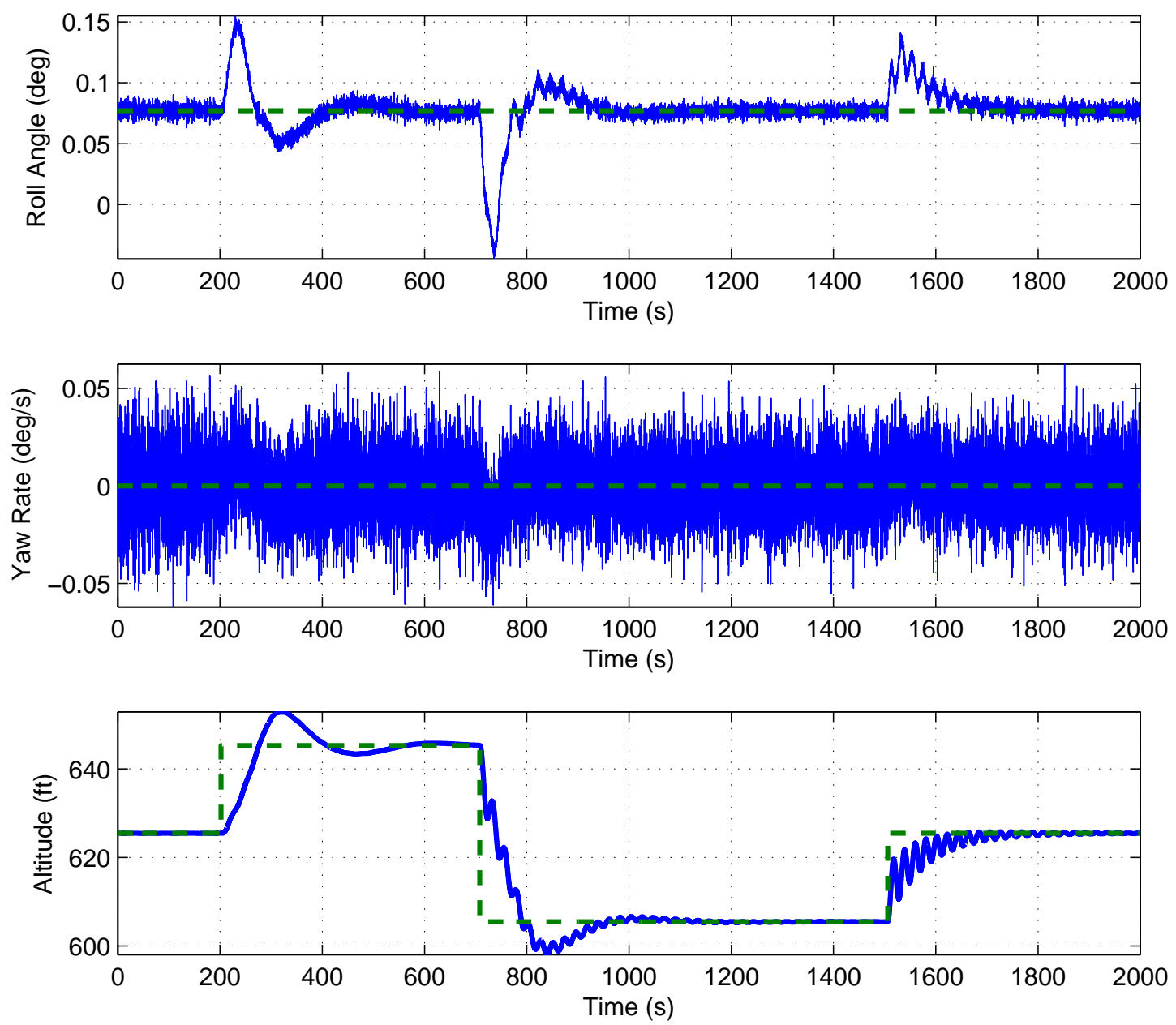

Figure 13: Aircraft response to doublet command as shown in Figure 12 under RCF adaptive control. The solid lines represent true aircraft response, while the dashed lines represent commanded performance. 
Control Surfaces
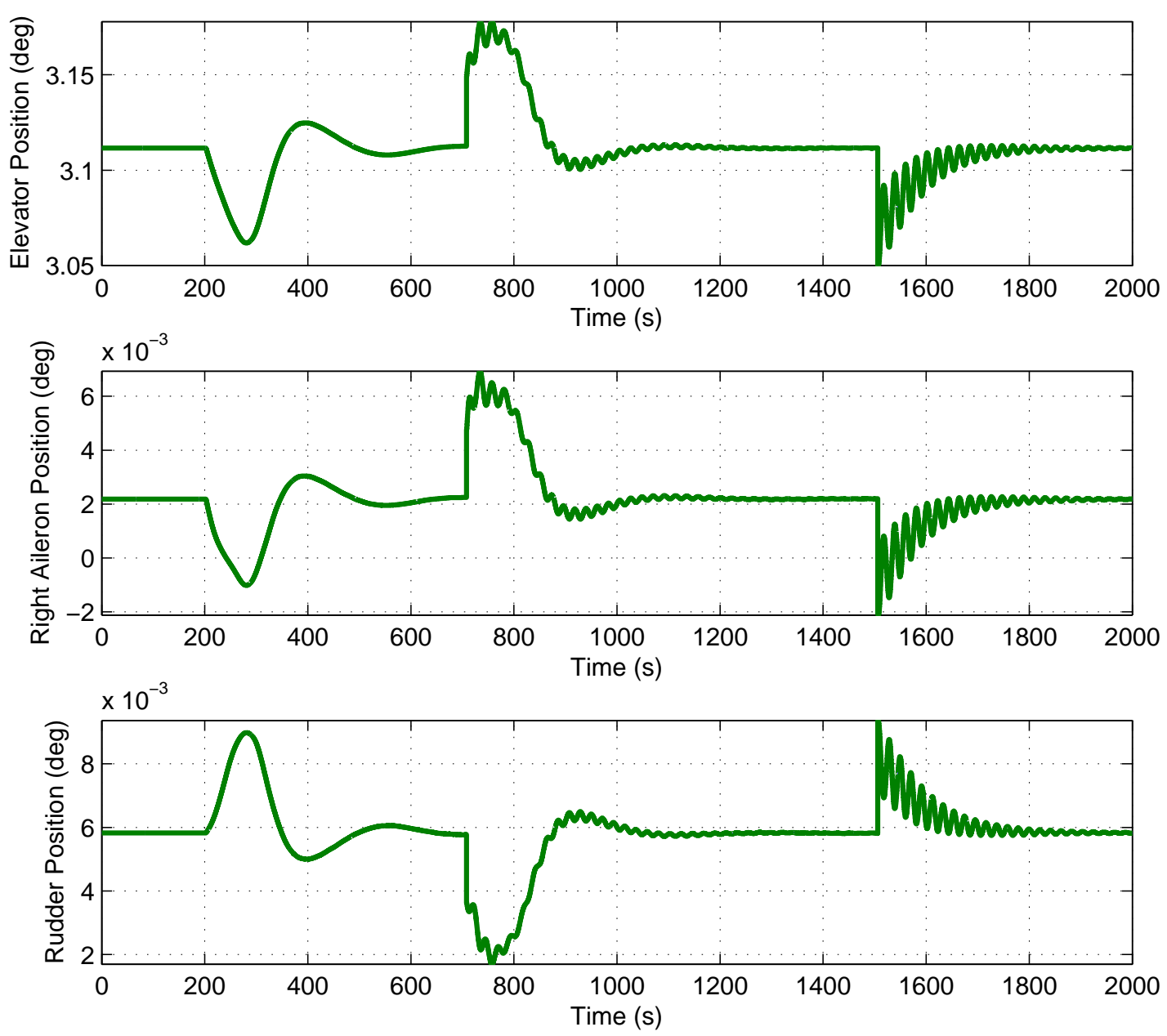

Figure 14: Aircraft control surface deflections due to RCF adaptive control in response to the altitude doublet command shown in Figure 12. 


\section{Performance Variables}
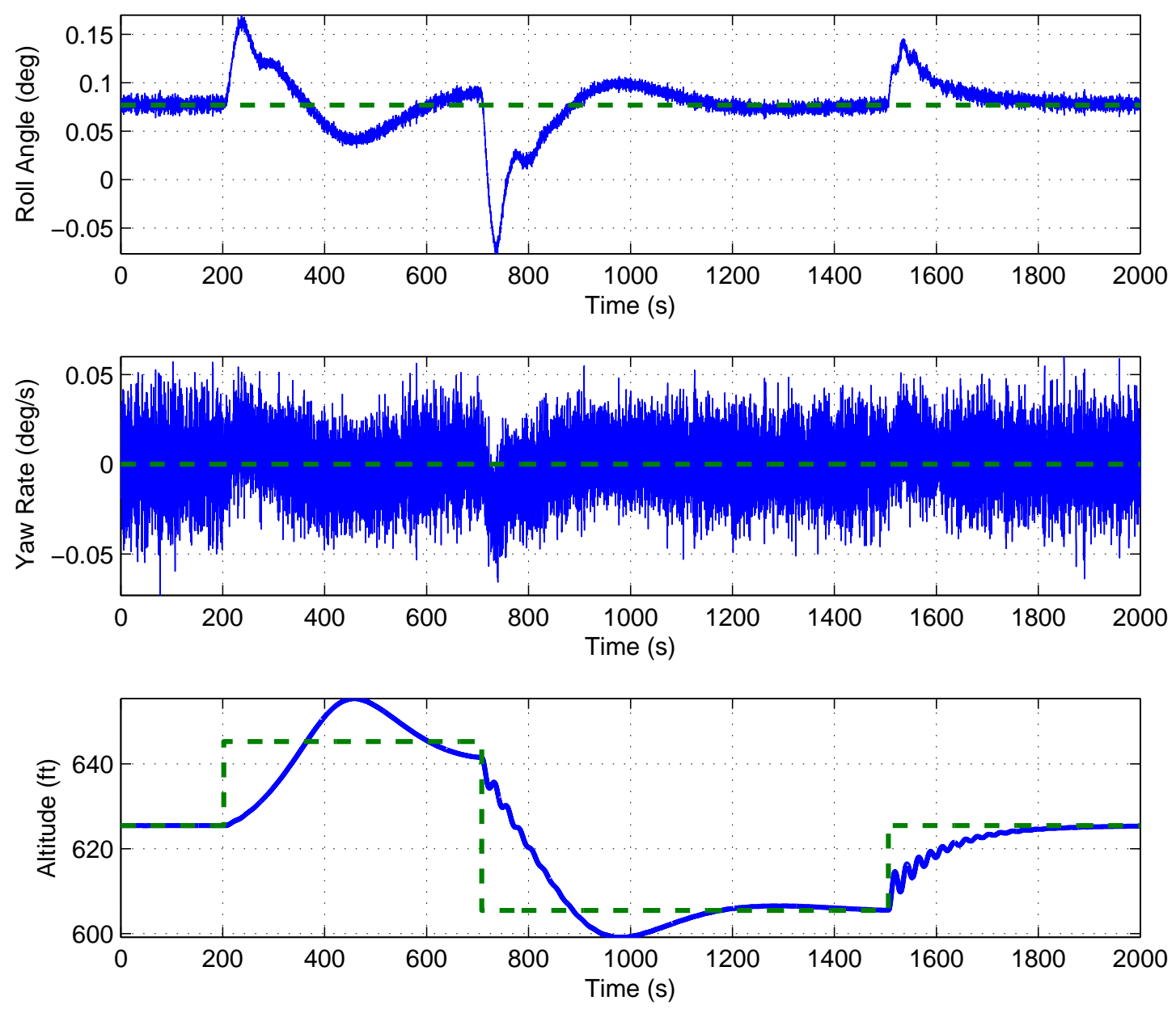

Figure 15: Aircraft response under RCF adaptive control to altitude doublet command as shown in Figure 12 with a $75 \%$ reduction in elevator effectiveness. The solid lines represent true aircraft response, while the dashed lines represent commanded performance. 


\section{Control Surfaces}
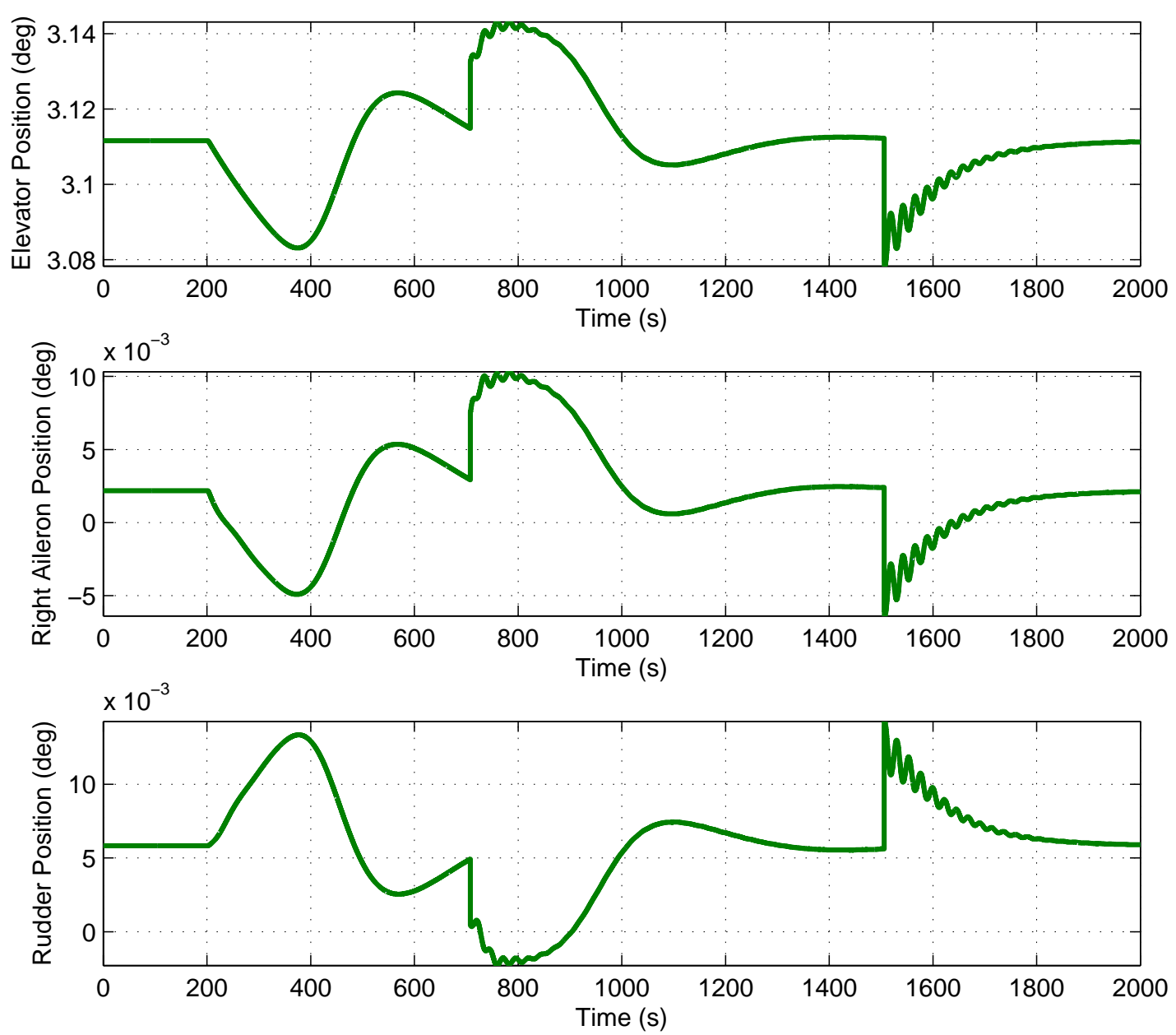

Figure 16: Aircraft control surface deflections due to RCF adaptive control in response to the altitude doublet command as shown in Figure 12 with a $75 \%$ reduction in elevator effectiveness. 